\title{
Alkene Oxyamination using Malonoyl Peroxides: Preparation of Pyrrolidines and Isoxazolidines
}

Carla Alamillo-Ferrer, ${ }^{\dagger}$ Jonathan M. Curle, ${ }^{\dagger}$ Stuart C. Davidson, ${ }^{\dagger}$ Simon C. C. Lucas,,$\stackrel{\dagger}{\dagger}$ Stephen J. Atkinson,

Matthew Campbell, ${ }^{\ddagger}$ Alan R. Kennedy ${ }^{\dagger}$ and Nicholas C. O. Tomkinson ${ }^{\dagger} * *$

${ }^{\dagger}$ WestCHEM, Department of Pure and Applied Chemistry, Thomas Graham Building, University of Strathclyde, 295 Cathedral Street, Glasgow, G1 1XL, United Kingdom.

†laxoSmithKline Medicines Research Centre, Gunnels Wood Road, Stevenage, SG1 2NY, United Kingdom.

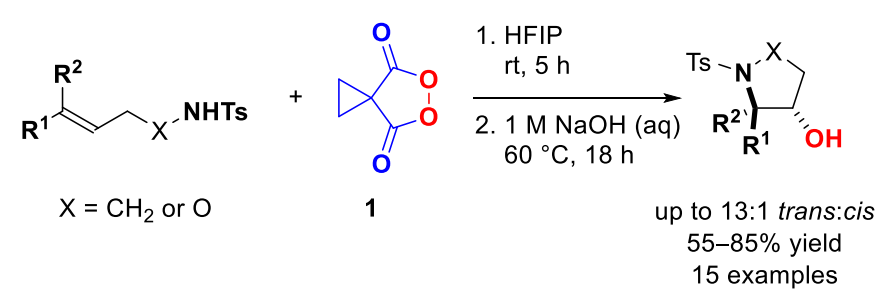

\begin{abstract}
Treatment of homoallylic $N$-tosyl amines or allylic $N$-tosyl hydroxylamines with 1.5 equiv of a malonoyl peroxide provides a stereoselective method to access functionalized pyrrolidines and isoxazolidines. This metal free alkene oxyamination proceeds in 55-85\% yield and up to 13:1 trans-selectivity. In addition, the relative stereochemistry of the oxygen and nitrogen substituents can be inverted through an oxidation/reduction sequence. Mechanistic investigations show a higher reactivity for hydroxyl nucleophiles over sulfonamide nucleophiles revealing a preference for dioxygenation over oxyamination.
\end{abstract}


The vicinal functionalization of alkenes is one of the most studied transformations in organic synthesis. This is particularly the case for the introduction of new carbon-heteroatom bonds generating up to two new stereogenic centers. For example, exquisite success has been achieved in dihydroxylation processes whereby the majority of alkene building blocks can be transformed stereo- and enantioselectively into the corresponding syn- or anti-diol using commercial reagents under mild and efficient conditions. ${ }^{1-3}$

Whilst significant efforts have been devoted to the di-oxygenation of alkenes, methods for oxyamination are substantially less developed, despite the accordant potential for the products in pharmaceutical and agrochemical research. ${ }^{4}$ The majority of methods developed for oxyamination involve the use of transition metal catalysts, including osmium, ${ }^{5,6}$ rhodium, ${ }^{7}$ palladium, ${ }^{8}$ copper ${ }^{9}$ and iron. ${ }^{10}$ Metal free methods have also been described and include both iodine $(0)^{11}$ and iodine(III) $)^{12}$ species as the reagent. Despite the extensive inroads made in the development of effective methods for the oxyamination of alkenes using these processes significant opportunities still exist in the area with regards to regio- and stereoselectivity along with substrate scope and the use of more environmentally benign catalysts, reagents, and reaction conditions.

We have recently shown that malonoyl peroxide $\mathbf{1}$ and its derivatives are versatile reagents for both the $s y n^{13}$ and anti-dioxygenation ${ }^{14,15}$ of alkenes. In the preparation of oxygen heterocycles transformations proceed through the reaction of the substrate alkene $\mathbf{2}$ with the malonoyl peroxide to give a dioxonium intermediate $3^{16}$ which can react stereoselectively in an intramolecular fashion with either an alcohol or a carboxylic acid to give the corresponding tetrahydrofuran or $\gamma$-lactone respectively (Scheme 1). Encouraged by the success of this work we sought to discover if the overall strategy could be applied to the preparation of nitrogen containing heterocycles through the reaction of alkenes tethered directly to a nitrogen nucleophile (e.g. 5). Within this paper we describe the development of a simple and effective intramolecular oxyamination procedure to deliver 2-hydroxypyrrolidine derivatives 7 in a stereoselective manner.

\section{Scheme 1. Intramolecular Oxidative Cyclizations}




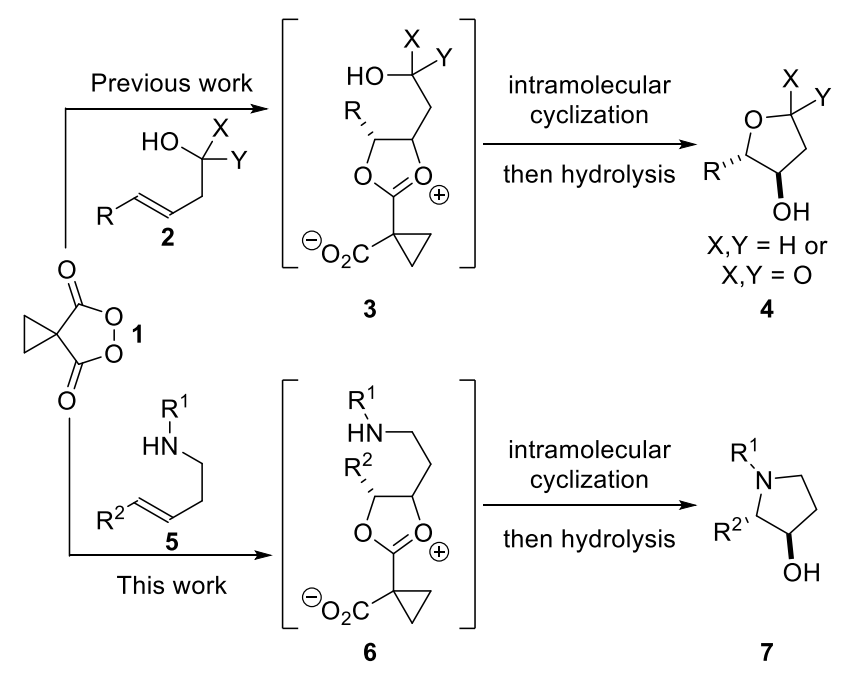

Our investigation began with the reaction of a homoallylic amine 8a $(\mathrm{R}=\mathrm{Ts})$ with malonoyl peroxide 1 (Table 1). Chlorinated solvents, which had proved effective in both the syn- and anti-dioxygenation of alkenes, ${ }^{13,14}$ led to slow consumption of starting materials with no clear indication of the desired pyrrolidine product 9a $(\mathrm{R}=\mathrm{Ts})$ (entries 1 and 2$)$. However, reaction of 8a with 1.5 equiv of malonoyl peroxide 1 at room temperature for $5 \mathrm{~h}$ in hexafluoroisopropanol (HFIP) followed by hydrolysis of the product led to the desired trans-3-hydroxypyrrolidine 9a in an excellent $69 \%$ isolated yield and a 1:9 cis:trans selectivity (entry 3). Drying the HFIP solvent over $3 \AA$ molecular sieves for $24 \mathrm{~h}$ prior to use provided the product in a similar yield and improved selectivity (entry $4 ; 71 \% ; 1: 13$ cis:trans). Interestingly, despite extensive efforts to induce similar reactivity with alternative amide $\mathbf{8 b}$ (entry 5), carbamate 8c and 8d (entries 6 and 7) and sulfonamide 8e (entry 8) substituents on the nitrogen nucleophile, similar reactivity patterns could not be observed upon complete consumption of starting material. In each of these transformations numerous products were present in the ${ }^{1} \mathrm{H}$ NMR of the crude reaction mixture with no clear evidence for the formation of the target pyrrolidines. This suggests that both the electronics and sterics of the nitrogen nucleophile are crucial to observing the desired reactivity and that $N$-tosylsulfonamides provide the environment required to promote reactivity. 
Table 1. Influence of Nitrogen Substituent

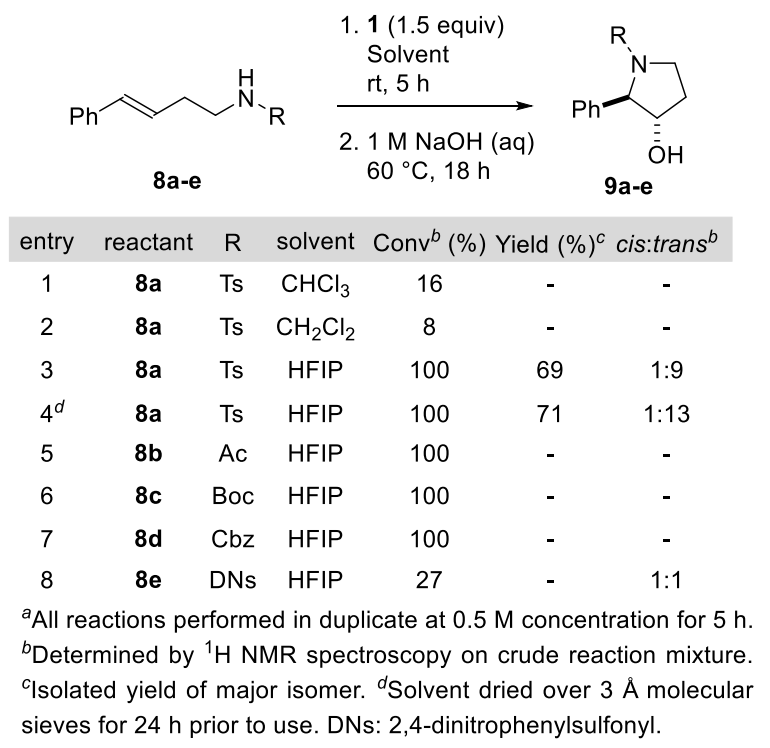

Confirmation of the structure of the cyclized pyrrolidine 10, prior to hydrolysis, came from single crystal X-ray crystallography (Figure 1). This structure clearly shows the trans-relationship between the phenyl group from the alkene substrate and the ester group from the malonoyl peroxide reagent. Overall, this provides a convenient, mild and effective method for the anti-oxyamination of alkenes.

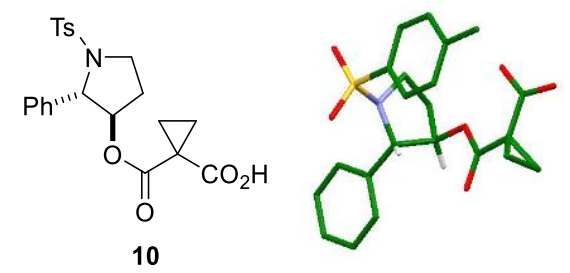

Figure 1. Single-crystal X-ray structure of pyrrolidine $\mathbf{1 0 .}$

Having established an efficient method for the oxyamination of $\mathbf{8 a}(\mathrm{R}=\mathrm{Ts})$ we went on to examine some of the scope of the process (Table 2). Substitution on all positions of the aromatic ring was tolerated with $p$ - (entry $2 ; 66 \% ; 1: 7$ cis:trans), $m$ - (entry 3;71\%; 1:6 cis:trans) and $o$-tolyl (entry 4; 72\%) substrates effectively undergoing the intramolecular oxyamination process. Chloride substitution was also well tolerated (entries 6 and 7), providing the products in up to $67 \%$ isolated yield which could readily be diversified. Alternative substitution on the aromatic ring that was also investigated included a $p$-phenyl substituent (entry 5; 72\%; 1:9 cis:trans) and an acetal group (entry 8; 55\%; 1:9 cis:trans). 


\section{Table 2. Substrate Scope for Oxyamination Procedure}
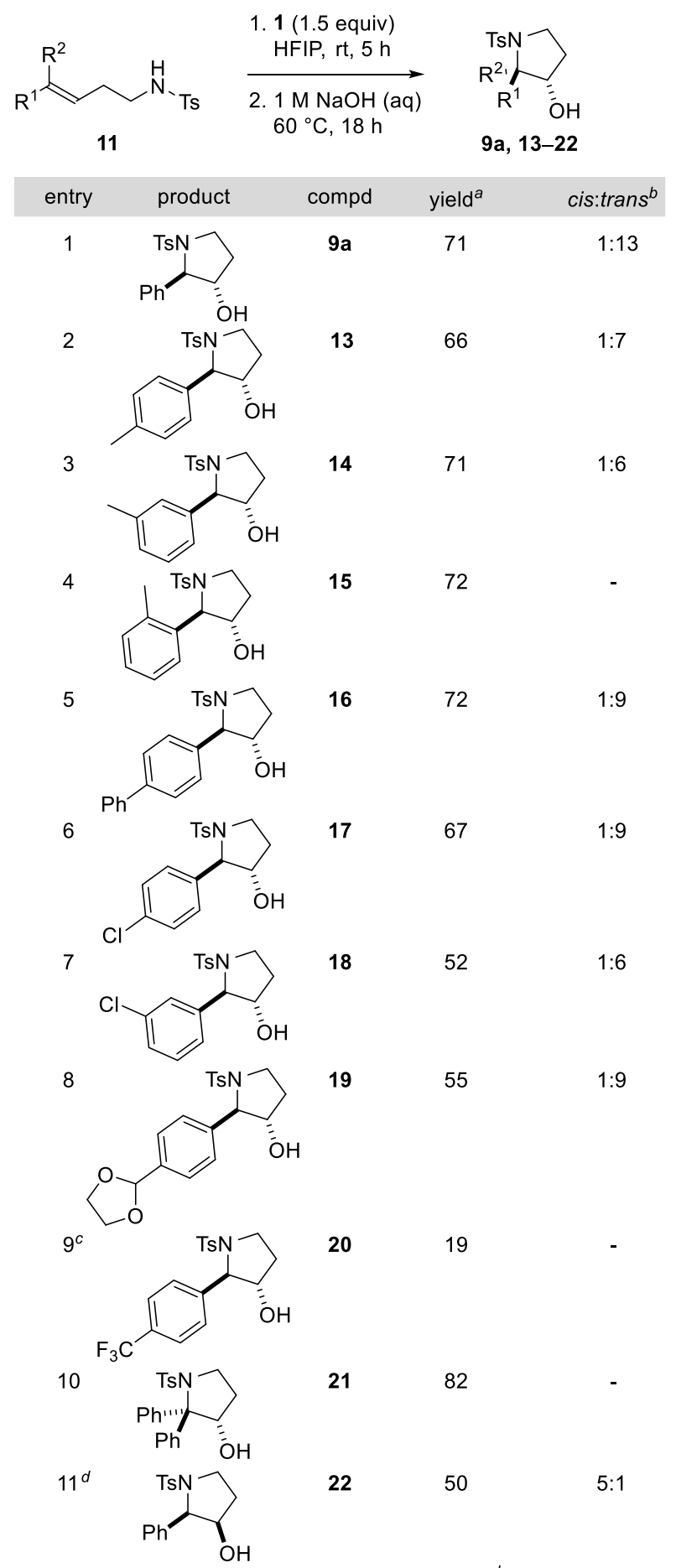

${ }^{a}$ Yields quoted are isolated yields of major isomer. ${ }^{b}$ Diastereoselectivity determined by ${ }^{1} \mathrm{H}$ NMR spectroscopy on the crude reaction mixture. ${ }^{c}$ Reaction conducted at $50{ }^{\circ} \mathrm{C}$ for $20 \mathrm{~h} .{ }^{d}$ Cis-alkene substrate used.

A limitation was encountered with the introduction of the strongly electron withdrawing trifluoromethyl substituent which was significantly slower than the other substrates examined. Conducting the 
reaction at a higher temperature of $50{ }^{\circ} \mathrm{C}$ for $20 \mathrm{~h}$ gave the product $\mathbf{2 0}$ in just $19 \%$ isolated yield (entry 9 ).

Whilst ${ }^{1} \mathrm{H}$ NMR spectroscopy supported the structure of $\mathbf{2 0}$ it was not possible to determine the stereoselectivity due to overlapping signals. Introduction of further substitution on the alkene substrate was also possible with the diphenyl-substituted pyrrolidine 21 being isolated in an excellent $82 \%$ yield (entry 10), providing the most efficient pyrrolidine synthesis examined within this study. Use of a $Z$-alkene substrate gave the cis-pyrrolidine product $\mathbf{2 2}$ as a 5:1 mixture of cis- and trans-isomers (entry 11).

Mechanistically we believe that the reaction is proceeding as outlined in Figure 2. Nucleophilic attack of the substituted alkene $\mathbf{2 3}$ on the peroxide $\mathbf{1}$ leads to $\mathbf{2 4}$ which cyclizes intramolecularly to give the dioxonium species $\mathbf{2 5}$ as defined previously for alkene dihydroxylation. ${ }^{16}$ Subsequent cyclization of the nitrogen nucleophile forms the pyrrolidine ring $\mathbf{2 7}$ with a trans-relationship of the newly formed C$\mathrm{O}$ and $\mathrm{C}-\mathrm{N}$ bonds. This mechanistic pathway is consistent with previous investigations into the reactivity of malonoyl peroxide $\mathbf{1}$ and accounts for the stereoselectivities observed within the process.

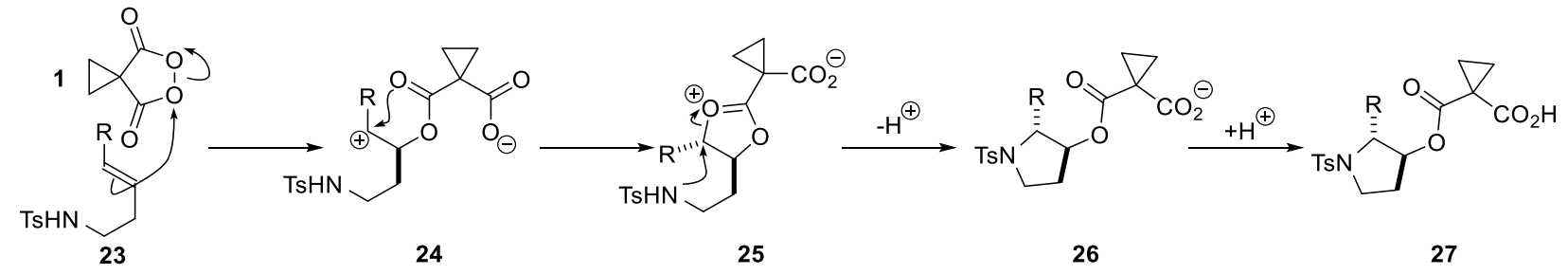

Figure 2. Proposed mechanistic pathway for the intramolecular oxyamination procedure.

In order to understand the mechanism of this transformation further we prepared the probe molecule 28, to ascertain if differences in nucleophilicity between heteroatoms could influence the outcome of the cyclization (Scheme 2). Compound $\mathbf{2 8}$ is a tetra-substituted alkene which contains both an alcohol and a sulfonamide nucleophile. Reaction of $\mathbf{2 8}$ under standard cyclization conditions followed by esterification of the crude product gave the tetrahydrofuran 29 (46\%). The pyrrolidine 30 was not detected by ${ }^{1} \mathrm{H}$ NMR spectroscopy on the crude reaction mixture. This shows that on formation of a dioxonium ion intermediate $\mathbf{3 2}$ the more nucleophilic pendant heteroatom reacts selectively through carbon atom A. In addition, we also isolated a small amount of the bicyclic compound $\mathbf{3 1}(\mathbf{7 \%})$ which results from direct cyclization of the oxygen heteroatom on carbon atom $\mathbf{B}$ of the dioxonium intermediate $\mathbf{3 2}$. We believe 
this product is formed due to the increased steric encumbrance of carbon atom $\mathbf{A}$, reducing the reactivity of this center.

\section{Scheme 2. Probe to Understand Reaction Mechanism}
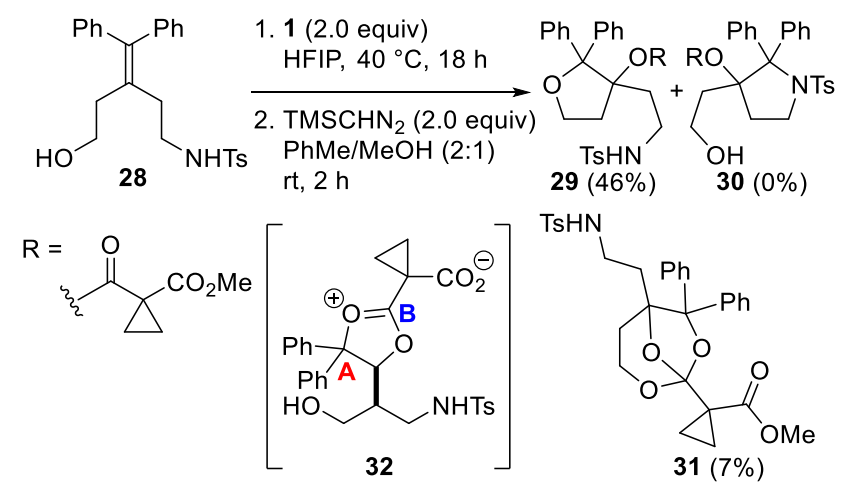

To expand the scope of the transformation to alternative nitrogen containing heterocycles we prepared the hydroxylamine derivatives $\mathbf{3 3}$ and $\mathbf{3 5}$ and reacted each with 1.5 equiv $\mathbf{1}$ (HFIP, $40{ }^{\circ} \mathrm{C}, 18 \mathrm{~h}$ ) (Scheme 3). The elevated temperature and extended reaction times necessary to bring about reaction suggest a reduced nucleophilicity of the nitrogen heteroatom within these substrates. After treatment of the crude reaction mixture with $\mathrm{TMSCHN}_{2}$ the isoxazolidines $34(80 \% ; 1: 10$ cis:trans) and $36(85 \% ; 1: 7$ cis:trans) were isolated after purification by column chromatography. This provides an efficient and highly stereoselective method for the preparation of the 4-hydroxyisoxazolidine framework from readily accessible alkene substrates.

\section{Scheme 3. Preparation of Isoxazolidines}

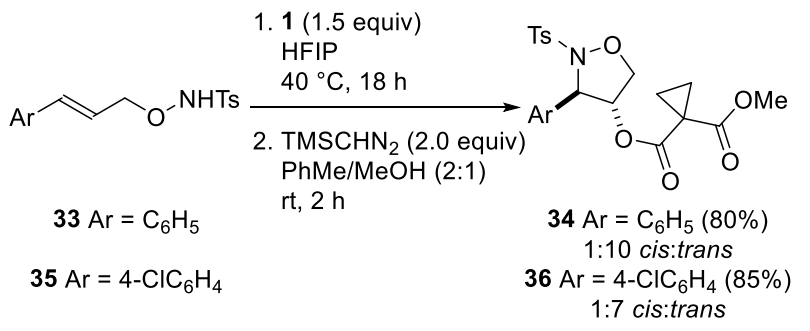

The structure of the isoxazolidine product $\mathbf{3 7}$, prior to esterification, was confirmed by single crystal X-ray crystallographic analysis (Figure 3). In contrast to the structure of the pyrrolidine analogue $\mathbf{1 0}$ the nitrogen atom adopts a tetrahedral geometry in this structure, with the isoxazolidine ring having a 
more puckered conformation. The apparent reduced nucleophilicity of the hydroxylamine derivatives $\mathbf{3 3}$ and $\mathbf{3 5}$ was surprising. Whilst we are unable to unequivocally explain this observation at present, the different structures of compounds $\mathbf{1 0}$ and $\mathbf{3 7}$ suggest that the conformation of the substrates may affect reactivity.
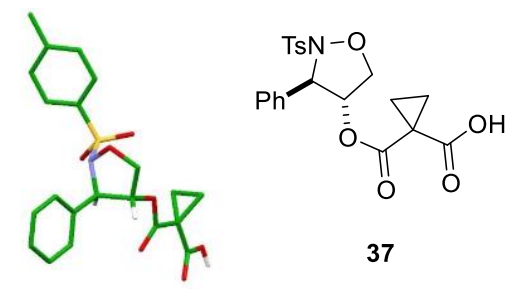

37

Figure 3. Single crystal X-ray structure of isoxazolidine 37.

Reaction of the 3-hydroxypyrrolidine 9a with IBX (3 equiv) in acetonitrile gave the pyrrolidinone 38 in $85 \%$ isolated yield (Scheme 4). Reduction of this ketone with DIBAL-H in THF at rt for $3 \mathrm{~h}$ gave the cis-substituted product 22 (6:1 cis:trans; 78\%). This sequence provides an alternative access to the diasteremeric pyrrolidine product $\mathbf{2 2}$ without independent preparation of the Z-alkene substrate enhancing the overall use of the process described and the diversity of structures accessed using this methodology.

\section{Scheme 4. Inverting Relative Stereochemistry of Pyrrolidine Ring}

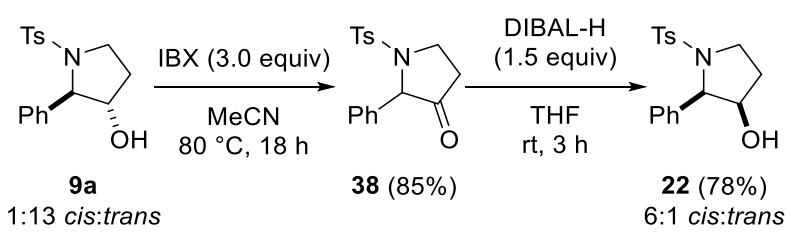

In summary, we have described a simple and effective method for the intramolecular oxyamination of alkenes. Through optimization of the nitrogen substituent we have shown that the oxyamination procedure can be induced at room temperature by treatment of an alkene substrate with 1.5 equiv of malonoyl peroxide 1 in HFIP. The product can be isolated in good yield and up to 13:1 trans:cis ratio. The related isoxazolidines can also be prepared from the appropriate hydroxylamine substrate. In addition, the relative stereochemistry of the two newly formed carbon-heteroatom bonds can be changed by preparation of the $Z$-alkene substrate or through a simple oxidation-reduction sequence, further expanding the scope of this useful transformation. A mechanistic probe showed there is a preference for reaction of the 
dioxonium intermediate with an alcohol over a sulfonamide nucleophile revealing a preference for dioxygenation over oxyamination. Current efforts are focused on developing an intermolecular variant of this procedure and we will report on our findings in the near future.

\section{Experimental Section}

Synthesis of malonoyl peroxide (1). ${ }^{15}$ Methane sulfonic acid $(30 \mathrm{~mL})$ was placed in a round bottomed flask equipped with a large magnetic stirrer bar and immersed in a bath of water at $22{ }^{\circ} \mathrm{C}$. Urea hydrogen peroxide $(9.8 \mathrm{~g}, 104.0 \mathrm{mmol})$ was added in a single portion and stirred for 30 seconds. Cyclopropane-1,1dicarboxylic acid $(5.0 \mathrm{~g}, 38.5 \mathrm{mmol})$ was added in a single portion and the reaction stirred vigorously for $18 \mathrm{~h}$. The reaction mixture was poured into a mixture of ice $(80 \mathrm{~g})$ and ethyl acetate $(100 \mathrm{~mL})$ and the layers separated. The aqueous layer was extracted with ethyl acetate $(2 \times 100 \mathrm{~mL})$ and the combined organics were washed with $\mathrm{NaHCO}_{3}(2 \times 50 \mathrm{~mL})$, brine $(20 \mathrm{~mL})$ and dried over $\mathrm{MgSO}_{4}$. Removal of the solvent under reduced pressure gave the desired peroxide $1(3.5 \mathrm{~g}, 27.3 \mathrm{mmol}, 71 \%)$. m.p. $90{ }^{\circ} \mathrm{C}$; IR (ATR) $/ \mathrm{cm}^{-1}: 1827,1798,1358 ;{ }^{1} \mathrm{H}$ NMR $\left(500 \mathrm{MHz}, \mathrm{CDCl}_{3}\right) \delta 2.10(\mathrm{~s}, 4 \mathrm{H}) ;{ }^{13} \mathrm{C} \mathrm{NMR}\left(126 \mathrm{MHz}, \mathrm{CDCl}_{3}\right)$ $\delta 172.3,23.7,19.9$.

Synthesis of 2-(But-3-en-1-yl)isoindoline-1,3-dione.${ }^{17} \mathrm{~A}$ dry three-neck flask was charged with potassium phthalimide (3.6 g, $19.2 \mathrm{mmol})$ in DMF (12 mL). 4-Bromobut-1-ene (1.5 mL, $14.8 \mathrm{mmol}$ ) was added and the mixture was stirred at reflux for $5 \mathrm{~h}$. The mixture was allowed to cool to room temperature, poured onto ice and extracted with $\mathrm{CH}_{2} \mathrm{Cl}_{2}(3 \times 50 \mathrm{~mL})$. The combined organics were washed with $0.2 \mathrm{M} \mathrm{KOH}$ (50 mL), $\mathrm{H}_{2} \mathrm{O}(50 \mathrm{~mL})$, dried over $\mathrm{MgSO}_{4}$, filtered and the solvent was removed by rotary evaporation to afford the title compound (2.8 $\mathrm{g}, 13.8 \mathrm{mmol}, 93 \%)$ as a brown solid which was used without further purification. m.p. $49-51{ }^{\circ} \mathrm{C}, \mathrm{Lit}^{18}\left[52-53{ }^{\circ} \mathrm{C}\right]$; IR (ATR)/cm ${ }^{-1}$ : 3075, 2974, 2939, 1772, 1705; ${ }^{1} \mathrm{H}$ NMR $\left(500 \mathrm{MHz}, \mathrm{CDCl}_{3}\right) \delta 7.85-7.81(\mathrm{~m}, 2 \mathrm{H}), 7.72-7.68(\mathrm{~m}, 2 \mathrm{H}), 5.79(\mathrm{ddt}, J=17.1,10.2,6.9 \mathrm{~Hz}, 1 \mathrm{H}), 5.06$ $(\mathrm{dd}, J=17.1,1.5 \mathrm{~Hz}, 1 \mathrm{H}), 5.01(\mathrm{~d}, J=10.2 \mathrm{~Hz}, 1 \mathrm{H}), 3.76(\mathrm{t}, J=7.1 \mathrm{~Hz}, 2 \mathrm{H}), 2.47-2.42(\mathrm{~m}, 2 \mathrm{H}) ;{ }^{13} \mathrm{C}$ NMR (126 MHz, $\left.\mathrm{CDCl}_{3}\right) \delta 168.5,134.6,134.0,132.3,123.3,117.7,37.5,33.0$; LRMS (ES + APCI) $m / z$ : $202.1[\mathrm{M}+\mathrm{H}]^{+}$. 
General Procedure 1: Heck Coupling. ${ }^{19}$ A dry three-neck flask fitted with a condenser was charged with aryl iodide (1.1 equiv) and $\mathrm{Et}_{3} \mathrm{~N}$ (2.0 equiv) in $\mathrm{MeCN}$ (0.05 M). 2-(But-3-en-1-yl)isoindoline-1,3-dione (1.0 equiv) was added followed by $\mathrm{P}(o \text {-tol })_{3}(10 \mathrm{~mol} \%)$ and $\mathrm{Pd}(\mathrm{OAc})_{2}(5 \mathrm{~mol} \%)$. The mixture was stirred at reflux until completion by TLC. The solution was allowed to cool to room temperature, passed through a plug of Celite ${ }^{\circledR}$ and the solvent was removed in vacuo. The crude residue was dissolved in EtOAc (100 $\mathrm{mL})$ and washed with $2 \mathrm{M} \mathrm{HCl}(\times 2), \mathrm{H}_{2} \mathrm{O}(\times 2)$, dried over $\mathrm{MgSO}_{4}$, filtered and the solvent was removed by rotary evaporation. Purification by silica gel flash column chromatography with hexane:EtOAc mixtures afforded the target compounds.

(E)-2-(4-Phenylbut-3-en-1-yl)isoindoline-1,3-dione. ${ }^{20}$ Phenyl iodide (1.7 mL, $\left.14.8 \mathrm{mmol}\right)$ and $\mathrm{Et}_{3} \mathrm{~N}(3.7$ $\mathrm{mL}, 26.8 \mathrm{mmol})$ were dissolved in $\mathrm{MeCN}(270 \mathrm{~mL})$ followed by addition of 2-(but-3-en-1-yl)isoindoline1,3-dione (2.7 g, $13.4 \mathrm{mmol}), \mathrm{P}(\mathrm{o} \text {-tol })_{3}(407 \mathrm{mg}, 1.3 \mathrm{mmol})$ and $\mathrm{Pd}(\mathrm{OAc})_{2}(150 \mathrm{mg}, 0.7 \mathrm{mmol})$ according to General Procedure 1 and the resulting mixture was heated at reflux for $16 \mathrm{~h}$. Purification by silica gel flash column chromatography (hexane:EtOAc 9:1) afforded the title compound (2.2 g, 7.9 mmol, 60\%) as a white solid. m.p. $138-139{ }^{\circ} \mathrm{C}, \mathrm{Lit}^{20}\left[133.5-135.5^{\circ} \mathrm{C}\right]$; IR (ATR)/cm ${ }^{-1}: 3054,3025,2935,1695 ;{ }^{1} \mathrm{H}$ $\operatorname{NMR}\left(500 \mathrm{MHz}, \mathrm{CDCl}_{3}\right) \delta$ 7.84-7.82 (m, 2H), 7.70-7.69 (m, 2H), 7.30-7.27 (m, 4H), 7.20-7.18 (m, 1H), $6.43(\mathrm{~d}, J=15.8 \mathrm{~Hz}, 1 \mathrm{H}), 6.18(\mathrm{dt}, J=15.8,7.1 \mathrm{~Hz}, 1 \mathrm{H}), 3.85(\mathrm{t}, J=7.1 \mathrm{~Hz}, 2 \mathrm{H}), 2.63-2.59(\mathrm{~m}, 2 \mathrm{H})$; ${ }^{13} \mathrm{C}$ NMR $\left(126 \mathrm{MHz}, \mathrm{CDCl}_{3}\right) \delta 168.3,137.3,133.9,132.6,132.1,128.5,127.2,126.2,126.1,123.2,37.6,32.2$; LRMS (ES + APCI) $m / z: 278.0[\mathrm{M}+\mathrm{H}]^{+}$.

(E)-2-(4-p-Tolyl)but-3-en-1-yl)isoindoline-1,3-dione. ${ }^{21}$ 4-Iodotoluene (600 mg, $\left.2.7 \mathrm{mmol}\right)$ and Et $3 \mathrm{~N}(700$ $\mu \mathrm{L}, 5.0 \mathrm{mmol}$ ) were dissolved in $\mathrm{MeCN}(50 \mathrm{~mL}$ ) followed by addition of 2-(but-3-en-1-yl)isoindoline1,3-dione (500 mg, $2.5 \mathrm{mmol}), \mathrm{P}(o \text {-tol })_{3}(75 \mathrm{mg}, 0.3 \mathrm{mmol})$ and $\mathrm{Pd}(\mathrm{OAc})_{2}(28 \mathrm{mg}, 0.1 \mathrm{mmol})$ according to General Procedure 1 and the resulting mixture was heated at reflux for $24 \mathrm{~h}$. Purification by silica gel flash column chromatography (hexane:EtOAc 9:1) afforded the title compound (432 mg, $1.5 \mathrm{mmol}, 60 \%$ ) as a white solid. m.p. $121-122{ }^{\circ} \mathrm{C}$; IR (ATR)/ $\mathrm{cm}^{-1}: 3023,2922,2854,1708 ;{ }^{1} \mathrm{H} \mathrm{NMR}\left(500 \mathrm{MHz}, \mathrm{CDCl}_{3}\right)$ $\delta 7.84-7.81(\mathrm{~m}, 2 \mathrm{H}), 7.71-7.68(\mathrm{~m}, 2 \mathrm{H}), 7.19(\mathrm{~d}, J=8.0 \mathrm{~Hz}, 2 \mathrm{H}), 7.08(\mathrm{~d}, J=8.0 \mathrm{~Hz}, 2 \mathrm{H}), 6.40(\mathrm{~d}, J=$ 
$15.9 \mathrm{~Hz}, 1 \mathrm{H}), 6.12(\mathrm{dt}, J=15.9,7.2 \mathrm{~Hz}, 1 \mathrm{H}), 3.83(\mathrm{t}, J=7.2 \mathrm{~Hz}, 2 \mathrm{H}), 2.62-2.57(\mathrm{~m}, 2 \mathrm{H}), 2.31(\mathrm{~s}, 3 \mathrm{H})$;

${ }^{13} \mathrm{C} \mathrm{NMR}\left(126 \mathrm{MHz} \mathrm{CDCl}_{3}\right) \delta 168.5,137.1,134.6,134.0,132.6,132.3,129.3,126.2,125.2,123.4,37.8$, 32.4, 21.3; LRMS (ES + APCI) $m / z: 292.0[\mathrm{M}+\mathrm{H}]^{+}$.

(E)-2-(4-m-Tolyl)but-3-en-1-yl)isoindoline-1,3-dione. ${ }^{21}$ 3-Iodotoluene (350 $\left.\mu \mathrm{L}, 2.7 \mathrm{mmol}\right)$ and Et $3 \mathrm{~N}(700$ $\mu \mathrm{L}, 5.0 \mathrm{mmol}$ ) were dissolved in $\mathrm{MeCN}$ (50 mL) followed by addition of 2-(but-3-en-1-yl)isoindoline1,3-dione (500 mg, $2.5 \mathrm{mmol}), \mathrm{P}(o \text {-tol })_{3}(75 \mathrm{mg}, 0.3 \mathrm{mmol})$ and $\mathrm{Pd}(\mathrm{OAc})_{2}(28 \mathrm{mg}, 0.1 \mathrm{mmol})$ according to General Procedure 1 and the resulting mixture was heated at reflux for $20 \mathrm{~h}$. Purification by silica gel flash column chromatography (hexane:EtOAc 9:1) afforded the title compound (528 mg, $1.8 \mathrm{mmol}, 73 \%$ ) as a colourless oil. IR (ATR)/ $\mathrm{cm}^{-1}: 3055,2940,2857,1712 ;{ }^{1} \mathrm{H}$ NMR $\left(500 \mathrm{MHz}, \mathrm{CDCl}_{3}\right) \delta 7.84-7.82(\mathrm{~m}$, 2H), 7.70-7.68 (m, 2H), $7.16(\mathrm{t}, J=7.4 \mathrm{~Hz}, 1 \mathrm{H}), 7.11(\mathrm{~s}, 1 \mathrm{H}), 7.11-7.09(\mathrm{~m}, 1 \mathrm{H}), 7.01(\mathrm{~d}, J=7.4 \mathrm{~Hz}$, $1 \mathrm{H}), 6.40(\mathrm{~d}, J=15.8 \mathrm{~Hz}, 1 \mathrm{H}), 6.16(\mathrm{dt}, J=15.8,7.1 \mathrm{~Hz}, 1 \mathrm{H}), 3.84(\mathrm{t}, J=7.1 \mathrm{~Hz}, 2 \mathrm{H}), 2.63-2.58(\mathrm{~m}$, 2H), $2.31(\mathrm{~s}, 3 \mathrm{H}) ;{ }^{13} \mathrm{C}$ NMR $\left(126 \mathrm{MHz}, \mathrm{CDCl}_{3}\right) \delta 168.5,138.1,137.3,134.0,132.8,132.3,128.5,128.2$, 127.0, 126.0, 123.43, 123.37, 37.8, 32.4, 21.5; LRMS (ES + APCI) $m / z: 292.0[\mathrm{M}+\mathrm{H}]^{+}$.

(E)-2-(4-o-Tolyl)but-3-en-1-yl)isoindoline-1,3-dione. ${ }^{21}$ 2-Iodotoluene (350 $\left.\mu \mathrm{L}, 2.7 \mathrm{mmol}\right)$ and $\mathrm{Et}_{3} \mathrm{~N}(700$ $\mu \mathrm{L}, 5.0 \mathrm{mmol})$ were dissolved in $\mathrm{MeCN}(50 \mathrm{~mL})$ followed by addition of 2-(but-3-en-1-yl)isoindoline1,3-dione (500 mg, $2.5 \mathrm{mmol}), \mathrm{P}(o \text {-tol })_{3}(75 \mathrm{mg}, 0.3 \mathrm{mmol})$ and $\mathrm{Pd}(\mathrm{OAc})_{2}(28 \mathrm{mg}, 0.1 \mathrm{mmol})$ according to General Procedure 1 and the resulting mixture was heated at reflux for $16 \mathrm{~h}$. Purification by silica gel flash column chromatography (hexane:EtOAc 9:1) afforded the title compound (300 mg, $1.0 \mathrm{mmol}, 42 \%$ ) as a white solid. m.p. $132-134{ }^{\circ} \mathrm{C}$; IR (ATR)/ $\mathrm{cm}^{-1}: 3056,3017,2939,2855,1706 ;{ }^{1} \mathrm{H}$ NMR $(500 \mathrm{MHz}$, $\left.\mathrm{CDCl}_{3}\right) \delta 7.84-7.83(\mathrm{~m}, 2 \mathrm{H}), 7.71-7.69(\mathrm{~m}, 2 \mathrm{H}), 7.35(\mathrm{~d}, J=6.9 \mathrm{~Hz}, 1 \mathrm{H}), 7.14-7.06(\mathrm{~m}, 3 \mathrm{H}), 6.59(\mathrm{~d}, J$ $=15.6 \mathrm{~Hz}, 1 \mathrm{H}), 6.12(\mathrm{dt}, J=15.6,7.5 \mathrm{~Hz}, 1 \mathrm{H}), 3.86(\mathrm{t}, J=7.0 \mathrm{~Hz}, 2 \mathrm{H}), 2.66-2.61(\mathrm{~m}, 2 \mathrm{H}), 2.20(\mathrm{~s}, 3 \mathrm{H})$; ${ }^{13} \mathrm{C} \mathrm{NMR}\left(126 \mathrm{MHz}, \mathrm{CDCl}_{3}\right) \delta 168.5,136.6,135.2,134.1,132.3,130.8,130.2,127.7,127.3,126.2,126.0$, 123.4, 37.8, 32.6, 19.8; LRMS (ES + APCI) $m / z: 292.0[\mathrm{M}+\mathrm{H}]^{+}$.

(E)-2-(4-(4-Chlorophenyl)but-3-en-1-yl)isoindoline-1,3-dione. ${ }^{22}$ 1-Chloro-4-iodobenzene (1.3 g, 5.5 $\mathrm{mmol})$ and $\mathrm{Et}_{3} \mathrm{~N}(1.4 \mathrm{~mL}, 10.0 \mathrm{mmol})$ were dissolved in $\mathrm{MeCN}(100 \mathrm{~mL})$ followed by addition of 2-(but- 
3-en-1-yl)isoindoline-1,3-dione (1.0 g, $4.97 \mathrm{mmol}), \mathrm{P}(o \text {-tol })_{3}(151 \mathrm{mg}, 0.5 \mathrm{mmol})$ and $\mathrm{Pd}(\mathrm{OAc})_{2}(56 \mathrm{mg}$, $0.3 \mathrm{mmol}$ ) according to General Procedure 1 and the resulting mixture was heated at reflux for $42 \mathrm{~h}$. Purification by silica gel flash column chromatography (hexane:EtOAc 9:1) afforded the title compound $(1.1 \mathrm{~g}, 3.4 \mathrm{mmol}, 68 \%)$ as a white solid. m.p. $130-131{ }^{\circ} \mathrm{C}, \operatorname{Lit}^{22}\left[130.5-132.0{ }^{\circ} \mathrm{C}\right] ;$ IR (ATR)/cm ${ }^{-1}: 3058$, 3025, 3002, 2939, 1699; ${ }^{1} \mathrm{H}$ NMR (500 MHz, $\left.\mathrm{CDCl}_{3}\right) \delta$ 7.84-7.82 (m, 2H), 7.71-7.69 (m, 2H), 7.23 (d, $J$ $=8.8 \mathrm{~Hz}, 2 \mathrm{H}), 7.21(\mathrm{~d}, J=8.8 \mathrm{~Hz}, 2 \mathrm{H}), 6.37(\mathrm{~d}, J=15.8 \mathrm{~Hz}, 1 \mathrm{H}), 6.15(\mathrm{dt}, J=15.8,7.1 \mathrm{~Hz}, 1 \mathrm{H}), 3.84$ $(\mathrm{t}, J=7.1 \mathrm{~Hz}, 2 \mathrm{H}), 2.62-2.58(\mathrm{~m}, 2 \mathrm{H}) ;{ }^{13} \mathrm{C} \mathrm{NMR}\left(126 \mathrm{MHz}, \mathrm{CDCl}_{3}\right) \delta 168.5,135.9,134.1,133.0,132.2$, $131.5,128.8,127.5,127.1,123.4,37.6,32.4 ;$ LRMS (ES + APCI) $m / z: 328.9\left[\mathrm{M}+\mathrm{NH}_{4}\right]^{+}$.

(E)-2-(4-(3-Chlorophenyl)but-3-en-1-yl)isoindoline-1,3-dione. ${ }^{23}$ 1-Chloro-3-iodobenzene (2.0 g, 8.4 $\mathrm{mmol})$ and $\mathrm{Et}_{3} \mathrm{~N}(2.1 \mathrm{~mL}, 15.2 \mathrm{mmol})$ were dissolved in $\mathrm{MeCN}(150 \mathrm{~mL})$ followed by addition of 2-(but3-en-1-yl)isoindoline-1,3-dione (1.53 g, $7.6 \mathrm{mmol}), \mathrm{P}(\mathrm{o} \text {-tol })_{3}(232 \mathrm{mg}, 0.8 \mathrm{mmol})$ and $\mathrm{Pd}(\mathrm{OAc})_{2}(86 \mathrm{mg}$, $0.4 \mathrm{mmol}$ ) according to General Procedure 1 and the resulting mixture was heated at reflux for $48 \mathrm{~h}$. Purification by silica gel flash column chromatography (hexane:EtOAc 9:1) afforded the title compound (1.8 g, $5.8 \mathrm{mmol}, 76 \%$ ) as a brown solid. m.p. $106-108{ }^{\circ} \mathrm{C}$; IR (ATR) $/ \mathrm{cm}^{-1}: 3056,3025,2935,2854$, 1708; ${ }^{1} \mathrm{H}$ NMR (500 MHz, $\left.\mathrm{CDCl}_{3}\right) \delta$ 7.85-7.81 (m, 2H), 7.72-7.68 (m, 2H), $7.26(\mathrm{~s}, 1 \mathrm{H}), 7.21-7.14(\mathrm{~m}$, 3H), $6.36(\mathrm{~d}, J=15.8 \mathrm{~Hz}, 1 \mathrm{H}), 6.19(\mathrm{dt}, J=15.8,7.1 \mathrm{~Hz}, 1 \mathrm{H}), 3.84(\mathrm{t}, J=7.1 \mathrm{~Hz}, 2 \mathrm{H}), 2.63-2.59(\mathrm{~m}$, $2 \mathrm{H}) ;{ }^{13} \mathrm{C} \mathrm{NMR}\left(126 \mathrm{MHz}, \mathrm{CDCl}_{3}\right) \delta 168.5,139.2,134.6,134.1,132.2,131.5,129.8,128.0,127.3,126.3$, 124.5, 123.4, 37.6, 32.2; LRMS (ES + APCI) $m / z: 329.0\left[\mathrm{M}+\mathrm{NH}_{4}\right]^{+}$.

(E)-2-(4-([1,1'-Biphenyl]-4-yl)but-3-en-1-yl)isoindoline-1,3-dione. 4-Iodo-1,1'-biphenyl (1.7 g, 6.1 $\mathrm{mmol})$ and $\mathrm{Et}_{3} \mathrm{~N}(1.5 \mathrm{~mL}, 11.0 \mathrm{mmol})$ were dissolved in $\mathrm{MeCN}(110 \mathrm{~mL})$ followed by addition of 2-(but3-en-1-yl)isoindoline-1,3-dione (1.1 g, $5.5 \mathrm{mmol}), \mathrm{P}(o \text {-tol })_{3}(168 \mathrm{mg}, 0.6 \mathrm{mmol})$ and $\mathrm{Pd}(\mathrm{OAc})_{2}(62 \mathrm{mg}$, $0.3 \mathrm{mmol}$ ) according to General Procedure 1 and the resulting mixture was heated at reflux for $72 \mathrm{~h}$. Purification by silica gel flash column chromatography (hexane:EtOAc 8:2) afforded the title compound (0.8 g, $2.3 \mathrm{mmol}, 41 \%$ ) as a white solid. m.p. $198^{\circ} \mathrm{C}$ (decomp); IR (ATR) $/ \mathrm{cm}^{-1}: 2987,2935,2879,1720$; ${ }^{1} \mathrm{H}$ NMR (400 MHz, $\left.\mathrm{CDCl}_{3}\right) \delta$ 7.86-7.82 (m, 2H), 7.72-7.67 (m, 2H), 7.59-7.56 (m, 2H), 7.53-7.50 (m, 
2H), 7.44-7.40 (m, 2H), 7.38-7.36 (m, 2H), 7.35-7.30 (m, 1H), $6.47(\mathrm{~d}, J=15.8 \mathrm{~Hz}, 1 \mathrm{H}), 6.22(\mathrm{~d}, J=$ 15.8, 7.1 Hz, 1H), $3.87(\mathrm{t}, J=7.1 \mathrm{~Hz}, 2 \mathrm{H}), 2.67-2.61(\mathrm{~m}, 2 \mathrm{H}) ;{ }^{13} \mathrm{C} \mathrm{NMR}\left(101 \mathrm{MHz}, \mathrm{CDCl}_{3}\right) \delta 168.5$, $140.9,140.2,136.5,134.1,132.31,132.26,128.9,127.3,127.1,126.7,126.5,123.4,37.7,32.5$ (1 carbon missing); LRMS (ES + APCI) $m / z: 371.1\left[\mathrm{M}+\mathrm{NH}_{4}\right]^{+}$; HRMS (ESI-TOF) $m / z:[\mathrm{M}+\mathrm{H}]^{+}$calcd. for $\mathrm{C}_{24} \mathrm{H}_{20} \mathrm{NO}_{2}$ 354.1494; found 354.1491.

(E)-2-(4-(4-(1,3-Dioxalan-2-yl)phenyl)but-3-en-1-yl)isoindoline-1,3-dione. 2-(4-Iodophenyl)-1-dioxalane $(3.0 \mathrm{~g}, 10.9 \mathrm{mmol})$ and $\mathrm{Et}_{3} \mathrm{~N}(2.8 \mathrm{~mL}, 19.7 \mathrm{mmol})$ were dissolved in $\mathrm{MeCN}(200 \mathrm{~mL})$ followed by addition of 2-(but-3-en-1-yl)isoindoline-1,3-dione (2.0 g, $9.9 \mathrm{mmol}), \mathrm{P}(o \text {-tol })_{3}(301 \mathrm{mg}, 1.0 \mathrm{mmol})$ and $\mathrm{Pd}(\mathrm{OAc})_{2}(111 \mathrm{mg}, 0.5 \mathrm{mmol})$ according to General Procedure 1 and the resulting mixture was heated at reflux for $72 \mathrm{~h}$. Purification by silica gel flash column chromatography (hexane:EtOAc 9:1) afforded the title compound (1.6 g, $4.6 \mathrm{mmol}, 46 \%$ ) as a white solid. m.p. $120-121{ }^{\circ} \mathrm{C}$; IR (ATR)/cm ${ }^{-1}: 3026,2945$, 2883, 1706; ${ }^{1} \mathrm{H}$ NMR $\left(500 \mathrm{MHz}, \mathrm{CDCl}_{3}\right) \delta$ 7.84-7.80 (m, 2H), 7.71-7.67 (m, 2H), $7.38(\mathrm{~d}, J=8.2 \mathrm{~Hz}$ 2H), $7.30(\mathrm{~d}, J=8.2 \mathrm{~Hz}, 2 \mathrm{H}), 6.42(\mathrm{~d}, J=15.8 \mathrm{~Hz}, 1 \mathrm{H}), 6.19(\mathrm{dd}, J=15.8,7.1 \mathrm{~Hz}, 1 \mathrm{H}), 5.78(\mathrm{~s}, 1 \mathrm{H})$, $4.12-4.10(\mathrm{~m}, 2 \mathrm{H}), 4.04-4.00(\mathrm{~m}, 2 \mathrm{H}), 3.85(\mathrm{~d}, J=7.1 \mathrm{~Hz}, 2 \mathrm{H}), 2.63-2.59(\mathrm{~m}, 2 \mathrm{H}) ;{ }^{13} \mathrm{C} \mathrm{NMR}(101 \mathrm{MHz}$, $\left.\mathrm{CDCl}_{3}\right) \delta 168.5,138.4,136.9,134.1,132.4,132.2,127.0,126.8,126.3,123.4,103.7,65.4,37.7,32.4$ LRMS (ES + APCI) $m / z: 350.0[\mathrm{M}+\mathrm{H}]^{+}$; HRMS (ESI-TOF) $m / z:[\mathrm{M}+\mathrm{H}]^{+}$calcd. for $\mathrm{C}_{21} \mathrm{H}_{20} \mathrm{NO}_{4}$ 350.1392; found 350.1387 .

(E)-2-(4-(4-Trifluoromethylphenyl)but-3-en-1-yl)isoindoline-1,3-dione. ${ }^{24}$ 1-Iodo-4-(trifluoromethyl)benzene $(803 \mu \mathrm{L}, 5.5 \mathrm{mmol})$ and $\mathrm{Et}_{3} \mathrm{~N}(1.4 \mathrm{~mL}, 9.9 \mathrm{mmol})$ were dissolved in $\mathrm{MeCN}(100 \mathrm{~mL})$ followed by addition of 2-(but-3-en-1-yl)isoindoline-1,3-dione (1.0 g, $5.0 \mathrm{mmol}), \mathrm{P}(o-t o l) 3(151 \mathrm{mg}, 0.5 \mathrm{mmol})$ and $\mathrm{Pd}(\mathrm{OAc})_{2}(56 \mathrm{mg}, 0.3 \mathrm{mmol})$ according to General Procedure 1 and the resulting mixture was heated at reflux for $18 \mathrm{~h}$. Purification by silica gel flash column chromatography (hexane:EtOAc 9:1) afforded the title compound (1.2 g, $3.5 \mathrm{mmol}, 70 \%$ ) as a white solid. m.p. $150^{\circ} \mathrm{C}$ decomp; IR (ATR)/cm ${ }^{-1}: 2997,2866$, 1699; ${ }^{1} \mathrm{H}$ NMR $\left(500 \mathrm{MHz}, \mathrm{CDCl}_{3}\right) \delta$ 7.84-7.83 (m, 2H), 7.71-7.69 (m, 2H), 7.52 (d, $\left.J=8.1 \mathrm{~Hz}, 2 \mathrm{H}\right)$, $7.38(\mathrm{~d}, J=8.1 \mathrm{~Hz}, 2 \mathrm{H}), 6.45(\mathrm{~d}, J=15.8 \mathrm{~Hz}, 1 \mathrm{H}), 6.31-6.25(\mathrm{~m}, 1 \mathrm{H}), 3.87(\mathrm{t}, J=7.0 \mathrm{~Hz}, 2 \mathrm{H}), 2.66-$ 
$2.62(\mathrm{~m}, 2 \mathrm{H}) ;{ }^{13} \mathrm{C} \mathrm{NMR}\left(126 \mathrm{MHz}, \mathrm{CDCl}_{3}\right) \delta 168.5,140.8,134.2,134.1,132.2,131.5,129.3,127.7\left(J_{\mathrm{C}-\mathrm{F}}\right.$

$=348.5 \mathrm{~Hz}), 126.4,125.6\left(J_{\mathrm{C}-\mathrm{F}}=3.75 \mathrm{~Hz}\right), 123.4,37.5,32.4$; LRMS $(\mathrm{ES}+\mathrm{APCI}) \mathrm{m} / z: 346.0[\mathrm{M}+\mathrm{H}]^{+}$.

Synthesis of 2-(4,4-diphenylbut-3-en-1-yl)isoindoline-1,3-dione. ${ }^{1725-27}$ To a three-neck round-bottom flask dried and flushed with argon was added (3 hydroxypropyl)triphenylphosphonium bromide (2.6 g, $6.6 \mathrm{mmol})$ in anhydrous THF $(12 \mathrm{~mL})$. The resulting suspension was cooled to $-10^{\circ} \mathrm{C}$ using a $\mathrm{NaCl} / \mathrm{ice}$ bath. A $1 \mathrm{M}$ solution of $\operatorname{LiHMDS}(15 \mathrm{~mL})$ was added dropwise and the mixture was stirred at $-10{ }^{\circ} \mathrm{C}$ for $1 \mathrm{~h}$. Benzophenone $(1.0 \mathrm{~g}, 5.5 \mathrm{mmol})$ was then added dropwise and stirred at $-10{ }^{\circ} \mathrm{C}$ for $2 \mathrm{~h}$. The mixture was allowed to warm to $\mathrm{rt}$ and stirred for a further $18 \mathrm{~h}$. A saturated aqueous solution of $\mathrm{NH}_{4} \mathrm{Cl}(50 \mathrm{~mL})$ was then added. The organic layer was extracted with $\mathrm{Et}_{2} \mathrm{O}(2 \times 100 \mathrm{~mL})$, dried over $\mathrm{MgSO}_{4}$, filtered and the solvent was removed under reduced pressure. Purification by silica gel flash column chromatography (petroleum ether $\left(40-60^{\circ} \mathrm{C}\right)$ :EtOAc 6:4) afforded 4,4 diphenylbut 3-en-1-ol (1.0 g, $\left.4.5 \mathrm{mmol}, 81 \%\right)$ as a colourless oil. ${ }^{1} \mathrm{H}$ NMR (400 MHz, $\left.\mathrm{CDCl}_{3}\right) \delta$ 7.40-7.36 (m, 2H), 7.34-7.26 (m, 2H), 7.25-7.19 (m, 6H), $6.15(\mathrm{t}, J=7.5 \mathrm{~Hz}, 1 \mathrm{H}), 3.75(\mathrm{t}, J=6.5 \mathrm{~Hz}, 2 \mathrm{H}), 2.46-2.41(\mathrm{~m}, 2 \mathrm{H}), 1.39(\mathrm{bs}, 1 \mathrm{H}) ;{ }^{13} \mathrm{C} \mathrm{NMR}(101 \mathrm{MHz}$ $\left.\mathrm{CDCl}_{3}\right) \delta 144.3,142.4,139.8,129.9,128.2,128.1,127.2,127.1,125.2,62.6,33.3$.

To a solution of 4,4-diphenylbut-3-en-1-ol (300 mg, $1.3 \mathrm{mmol})$ in a 48\% solution of $\mathrm{HBr}(1.6 \mathrm{~mL})$ was added tetrabutylammonium bromide $(17 \mathrm{mg}, 0.1 \mathrm{mmol})$. The mixture was stirred at reflux for $18 \mathrm{~h}$. The mixture was then allowed to cool to rt and diluted with $\mathrm{CH}_{2} \mathrm{Cl}_{2}(10 \mathrm{~mL})$ and $\mathrm{H}_{2} \mathrm{O}(10 \mathrm{~mL})$. The organic layer was extracted with $\mathrm{CH}_{2} \mathrm{Cl}_{2}(2 \times 20 \mathrm{~mL})$, washed with $\mathrm{NaHCO}_{3}(50 \mathrm{~mL})$, brine $(50 \mathrm{~mL})$, dried over $\mathrm{MgSO}_{4}$ and filtered. The solvent was removed under reduced pressure to afford (4-bromobut-1-ene-1,1diyl)dibenzene (350 mg, $1.2 \mathrm{mmol}, 91 \%)$ as a yellow oil. ${ }^{1} \mathrm{H}$ NMR (400 $\left.\mathrm{MHz}, \mathrm{CDCl}_{3}\right) \delta 7.44-7.27(\mathrm{~m}$, 5H), 7.25-7.22 (m, 3H), 7.19-7.17 (m, 2H), $6.09(\mathrm{t}, J=7.3 \mathrm{~Hz}, 1 \mathrm{H}), 3.43(\mathrm{t}, J=7.0 \mathrm{~Hz}, 2 \mathrm{H}), 2.71-2.66$ $(\mathrm{m}, 2 \mathrm{H}) ;{ }^{13} \mathrm{C} \mathrm{NMR}\left(101 \mathrm{MHz}, \mathrm{CDCl}_{3}\right) \delta 144.3,142.1,139.6,129.7,128.3,128.1,127.29,127.26,125.7$, $32.9,32.6$.

A dry three-neck flask was charged with potassium phthalimide (360 mg, $1.9 \mathrm{mmol})$ in anhydrous DMF $(10 \mathrm{~mL})$. (4-bromobut-1-ene-1,1 diyl)dibenzene $(558 \mathrm{mg}, 1.9 \mathrm{mmol})$ was added and mixture was stirrer at 
reflux for $18 \mathrm{~h}$. The mixture was allowed to cool to $\mathrm{rt}$, poured into ice and extracted with $\mathrm{CH}_{2} \mathrm{Cl}_{2}(3 \times 50$ $\mathrm{mL})$. The organics were washed with $0.2 \mathrm{M} \mathrm{KOH}(50 \mathrm{~mL}), \mathrm{H}_{2} \mathrm{O}(50 \mathrm{~mL})$, dried over $\mathrm{MgSO}_{4}$, filtered and the solvent was removed under reduced pressure. Purification by silica gel flash column chromatography (petroleum ether $\left(40-60^{\circ} \mathrm{C}\right)$ :EtOAc 9:1) afforded the title compound $(600 \mathrm{mg}, 1.7 \mathrm{mmol}, 88 \%)$ as a white solid. m.p. $120-121{ }^{\circ} \mathrm{C}, \operatorname{Lit}^{28}\left[119-120{ }^{\circ} \mathrm{C}\right]$; IR (ATR)/cm ${ }^{-1}: 2967,2921,2908,2872,1718,1701 ;{ }^{1} \mathrm{H}$ $\operatorname{NMR}\left(400 \mathrm{MHz}, \mathrm{CDCl}_{3}\right) \delta$ 7.83-7.78 (m, 2H), 7.71-7.66 (m, 2H), 7.27-7.18 (m, 8H), 7.02-6.99 (m, 2H), $6.07(\mathrm{t}, J=7.6 \mathrm{~Hz}, 1 \mathrm{H}), 3.81-3.78(\mathrm{~m}, 2 \mathrm{H}), 2.57-2.52(\mathrm{~m}, 2 \mathrm{H}) ;{ }^{13} \mathrm{C} \mathrm{NMR}\left(101 \mathrm{MHz}, \mathrm{CDCl}_{3}\right) \delta 168.3$, $144.5,142.5,139.6,134.0,132.3,129.8,128.3,128.2,127.5,127.2,125.2,123.3,37.8,29.0$ (1 carbon missing); LRMS (ES + APCI) $m / z: 354.0[\mathrm{M}+\mathrm{H}]^{+}$.

General Procedure 2: Removal of phthalimide and tosylation. ${ }^{29}$ To a solution of the (E)-2-(4-arylbut-3en-1-yl)isoindoline-1,3-dione (1.0 equiv) in $\mathrm{EtOH}(0.3 \mathrm{M})$ was added hydrazine monohydrate (2.0 equiv). The mixture was stirred at room temperature for $10 \mathrm{~min}$ and at reflux for a further 30 minutes. After cooling to room temperature a $2 \mathrm{M}$ solution of $\mathrm{NaOH}$ was added $(50 \mathrm{~mL})$. The solvent was evaporated before extracting the organics with EtOAc $(3 \times 50 \mathrm{~mL})$ and drying over $\mathrm{MgSO}_{4}$. The solvent was removed by rotary evaporation to give the free amine compound which was used without further purification. The crude reaction mixture was dissolved in anhydrous $\mathrm{CH}_{2} \mathrm{Cl}_{2}(0.3 \mathrm{M})$ under an argon atmosphere and $\mathrm{Et}_{3} \mathrm{~N}$ (1.5 equiv) was added. The mixture was cooled to $0{ }^{\circ} \mathrm{C}$, and $p$-toluenesulfonyl chloride (1.0 equiv) and $\operatorname{DMAP}(0.3$ equiv $)$ were added in one portion. The mixture was stirred at room temperature for $24 \mathrm{~h}$. The solution was diluted with $\mathrm{CH}_{2} \mathrm{Cl}_{2}$ and washed with $2 \mathrm{M} \mathrm{HCl}(100 \mathrm{~mL})$ and brine $(100 \mathrm{~mL})$. The organics were dried over $\mathrm{MgSO}_{4}$ and the solvent was removed by rotary evaporation to give a crude material which was purified by silica gel chromatography eluting with hexane:EtOAc mixtures to afford the tosylated compound.

(E)-4-Methyl-N-(4-phenylbut-3-en-1-yl)benzenesulfonamide (8a). ${ }^{29}$ To a solution of (E)-2-(4-phenylbut3-en-1-yl)isoindoline-1,3-dione $(2.2 \mathrm{~g}, 7.4 \mathrm{mmol})$ in EtOH $(32 \mathrm{~mL})$ was added hydrazine monohydrate (771 $\mu \mathrm{L}, 15.8 \mathrm{mmol})$ according to General Procedure 2 to give $(E)$-4-phenylbut-3-en-1-amine. 
To a solution of (E)-4-phenylbut-3-en-1-amine (1.2 g, $7.9 \mathrm{mmol})$ in $\mathrm{CH}_{2} \mathrm{Cl}_{2}(65 \mathrm{~mL})$ was added $\mathrm{Et}_{3} \mathrm{~N}(2.3$ $\mathrm{mL}, 16.2 \mathrm{mmol})$ followed by $p$-TsCl $(1.8 \mathrm{~g}, 9.8 \mathrm{mmol})$ and DMAP $(0.6 \mathrm{~g}, 4.9 \mathrm{mmol})$ according to General Procedure 2. Purification by silica gel flash column chromatography (hexane:EtOAc 9:1) afforded the title compound 8a (1.8 g, $6.0 \mathrm{mmol}, 74 \%)$ as a white solid. m.p. $52-54{ }^{\circ} \mathrm{C}$; IR (ATR)/cm ${ }^{-1}: 3272,3058$, 3023, 2922; ${ }^{1} \mathrm{H}$ NMR (500 MHz, $\left.\mathrm{CDCl}_{3}\right) \delta 7.75(\mathrm{~d}, J=8.2 \mathrm{~Hz}, 2 \mathrm{H}), 7.31-7.27(\mathrm{~m}, 6 \mathrm{H}), 7.24-7.21(\mathrm{~m}$, 1H), $6.36(\mathrm{~d}, J=15.9 \mathrm{~Hz}, 1 \mathrm{H}), 5.98(\mathrm{dt}, J=15.9,7.1 \mathrm{~Hz}, 1 \mathrm{H}), 4.43(\mathrm{bs}, 1 \mathrm{H}), 3.13-3.09(\mathrm{~m}, 2 \mathrm{H}), 2.43$ (s, 3H), 2.39-2.35 (m, 2H); ${ }^{13} \mathrm{C}$ NMR (126 MHz, $\left.\mathrm{CDCl}_{3}\right) \delta 143.6,137.2,136.9,133.5,129.9,128.7,127.7$, 127.3, 126.3, 125.6, 42.7, 33.2, 21.7; LRMS (ES + APCI) m/z: $319.1\left[\mathrm{M}+\mathrm{NH}_{4}\right]^{+}$.

(E)-4-Methyl-N-(4-(p-tolyl)but-3-en-1-yl)benzenesulfonamide. ${ }^{30}$ To a solution of (E)-2-(4-(p-tolyl)but-3en-1-yl)isoindoline-1,3-dione $(430 \mathrm{mg}, 1.5 \mathrm{mmol})$ in $\mathrm{EtOH}(6.0 \mathrm{~mL})$ was added hydrazine monohydrate $(143 \mu \mathrm{L}, 3.0 \mathrm{mmol})$ according to General Procedure 2 to give $(E)-4$-( $p$-tolylbut)-3-en-1-amine.

To a solution of $(E)-4$-(p-tolylbut)-3-en-1-amine (165 mg, $1.0 \mathrm{mmol})$ in $\mathrm{CH}_{2} \mathrm{Cl}_{2}(4.0 \mathrm{~mL})$ was added $\mathrm{Et}_{3} \mathrm{~N}$ $(210 \mu \mathrm{L}, 1.5 \mathrm{mmol})$ followed by $p-\mathrm{TsCl}(190 \mathrm{mg}, 1.2 \mathrm{mmol})$ and DMAP (37 $\mathrm{mg}, 0.3 \mathrm{mmol})$ according to General Procedure 2. Purification by silica gel flash column chromatography (hexane:EtOAc 8:2) afforded the title compound (192 mg, $0.6 \mathrm{mmol}, 61 \%$ ) as a white solid. m.p. $82-84{ }^{\circ} \mathrm{C}$; IR (ATR) $/ \mathrm{cm}^{-1}$ : 3276, 3047, 3017, 2939; ${ }^{1} \mathrm{H}$ NMR $\left(500 \mathrm{MHz}, \mathrm{CDCl}_{3}\right) \delta 7.74(\mathrm{~d}, J=8.2 \mathrm{~Hz}, 2 \mathrm{H}), 7.29(\mathrm{~d}, J=8.2 \mathrm{~Hz}, 2 \mathrm{H})$, $7.17(\mathrm{~d}, J=8.0 \mathrm{~Hz}, 2 \mathrm{H}), 7.10(\mathrm{~d}, J=8.0 \mathrm{~Hz}, 2 \mathrm{H}), 6.32(\mathrm{~d}, J=15.8 \mathrm{~Hz}, 1 \mathrm{H}), 5.97(\mathrm{dt}, J=15.8,7.1 \mathrm{~Hz}$, $1 \mathrm{H}), 4.37(\mathrm{t}, J=5.4 \mathrm{~Hz}, 1 \mathrm{H}), 3.12-3.08(\mathrm{~m}, 2 \mathrm{H}), 2.43(\mathrm{~s}, 3 \mathrm{H}), 2.37-2.34(\mathrm{~m}, 2 \mathrm{H}), 2.33(\mathrm{~s}, 3 \mathrm{H}) ;{ }^{13} \mathrm{C} \mathrm{NMR}$ $\left(126 \mathrm{MHz} \mathrm{CDCl}_{3}\right) \delta 143.6,137.5,137.2,134.1,133.4,129.9,129.4,127.3,126.2,124.5,42.7,33.1,21.7$, 21.3; LRMS (ES + APCI) $m / z: 316.0[\mathrm{M}+\mathrm{H}]^{+}$.

(E)-4-Methyl-N-(4-(m-tolyl)but-3-en-1-yl)benzenesulfonamide. ${ }^{21}$ To a solution of (E)-2-(4-(m-tolyl)but3-en-1-yl)isoindoline-1,3-dione (340 mg, $1.2 \mathrm{mmol})$ in EtOH (4.5 mL) was added hydrazine monohydrate $(113 \mu \mathrm{L}, 2.3 \mathrm{mmol})$ according to General Procedure 2 to give $(E)-4-(m$-tolylbut $)-3$-en-1-amine.

To a solution of $(E)-4$-( $m$-tolylbut)-3-en-1-amine (189 mg, $1.2 \mathrm{mmol})$ in $\mathrm{CH}_{2} \mathrm{Cl}_{2}(4.0 \mathrm{~mL})$ was added $\mathrm{Et}_{3} \mathrm{~N}$ (245 $\mu \mathrm{L}, 1.8 \mathrm{mmol})$ followed by $p-\mathrm{TsCl}(223 \mathrm{mg}, 1.2 \mathrm{mmol})$ and DMAP (43 $\mathrm{mg}, 0.4 \mathrm{mmol})$ according to 
General Procedure 2. Purification by silica gel flash column chromatography (hexane:EtOAc 8:2) afforded the title compound (145 mg, $0.5 \mathrm{mmol}, 39 \%$ ) as a colourless oil. IR (ATR)/ $\mathrm{cm}^{-1}: 3277,3023,2919$, 2861; ${ }^{1} \mathrm{H}$ NMR $\left(500 \mathrm{MHz}, \mathrm{CDCl}_{3}\right) \delta 7.75(\mathrm{~d}, J=8.2 \mathrm{~Hz}, 2 \mathrm{H}), 7.29(\mathrm{~d}, J=8.2 \mathrm{~Hz}, 2 \mathrm{H}), 7.18(\mathrm{t}, J=7.6$ Hz, 1H), $7.10(\mathrm{~s}, 1 \mathrm{H}), 7.08(\mathrm{~d}, J=7.6 \mathrm{~Hz}, 1 \mathrm{H}), 7.04(\mathrm{~d}, J=7.6 \mathrm{~Hz}, 1 \mathrm{H}), 6.33(\mathrm{~d}, J=15.8 \mathrm{~Hz}, 1 \mathrm{H}), 5.97$ $(\mathrm{dt}, J=15.8,7.0 \mathrm{~Hz}, 1 \mathrm{H}), 4.66(\mathrm{t}, J=5.8 \mathrm{~Hz}, 1 \mathrm{H}), 3.11-3.07(\mathrm{~m}, 2 \mathrm{H}), 2.42(\mathrm{~s}, 3 \mathrm{H}), 2.38-2.33(\mathrm{~m}, 2 \mathrm{H})$, $2.33(\mathrm{~s}, 3 \mathrm{H}) ;{ }^{13} \mathrm{C} \mathrm{NMR}\left(126 \mathrm{MHz}, \mathrm{CDCl}_{3}\right) \delta 143.5,138.2,137.1,136.9,133.4,129.8,128.6,128.4,127.3$, 127.0, 125.5, 123.4, 42.7, 33.1, 21.6, 21.5; LRMS (ES + APCI) $m / z: 316.0[\mathrm{M}+\mathrm{H}]^{+}$.

(E)-4-Methyl-N-(4-(o-tolyl)but-3-en-1-yl)benzenesulfonamide. ${ }^{21}$ To a solution of (E)-2-(4-(o-tolyl)but-3en-1-yl)isoindoline-1,3-dione $(275 \mathrm{mg}, 0.9 \mathrm{mmol})$ in EtOH $(3.6 \mathrm{~mL})$ was added hydrazine monohydrate (92 $\mu \mathrm{L}, 1.9 \mathrm{mmol})$ according to General Procedure 2 to give $(E)-4-(o$-tolylbut)-3-en-1-amine.

To a solution of $(E)$-4-(o-tolylbut)-3-en-1-amine $(151 \mathrm{mg}, 0.9 \mathrm{mmol})$ in $\mathrm{CH}_{2} \mathrm{Cl}_{2}(4.0 \mathrm{~mL})$ was added $\mathrm{Et}_{3} \mathrm{~N}$ $(200 \mu \mathrm{L}, 1.4 \mathrm{mmol})$ followed by $p$-TsCl $(179 \mathrm{mg}, 0.9 \mathrm{mmol})$ and DMAP (35 mg, $0.3 \mathrm{mmol})$ according to General Procedure 2. Purification by silica gel flash column chromatography (hexane:EtOAc 8:2) afforded the title compound (64 mg, $0.2 \mathrm{mmol}, 22 \%$ ) as a colourless oil. IR (ATR)/ $\mathrm{cm}^{-1}: 3272,3021,2922$, 2865; ${ }^{1} \mathrm{H}$ NMR $\left(500 \mathrm{MHz}, \mathrm{CDCl}_{3}\right) \delta 7.76(\mathrm{~d}, J=8.1 \mathrm{~Hz}, 2 \mathrm{H}), 7.32-7.31(\mathrm{~m}, 1 \mathrm{H}), 7.28(\mathrm{~d}, J=8.1 \mathrm{~Hz}$, 2H), 7.15-7.11 (m, 3H), $6.58(\mathrm{~d}, J=15.7 \mathrm{~Hz}, 1 \mathrm{H}), 5.86(\mathrm{dt}, J=15.7,7.1 \mathrm{~Hz}, 1 \mathrm{H}), 4.65(\mathrm{bs}, 1 \mathrm{H}), 3.11(\mathrm{t}$, $J=6.7 \mathrm{~Hz}, 2 \mathrm{H}), 2.42(\mathrm{~s}, 3 \mathrm{H}), 2.42-2.37(\mathrm{~m}, 2 \mathrm{H}), 2.30(\mathrm{~s}, 3 \mathrm{H}) ;{ }^{13} \mathrm{C} \mathrm{NMR}\left(126 \mathrm{MHz}, \mathrm{CDCl}_{3}\right) \delta 143.6$, 137.2, 136.1, 135.2, 131.2, 130.4, 129.8, 127.5, 127.4, 127.0, 126.2, 125.6, 42.8, 33.4, 21.6, 19.9; LRMS $(\mathrm{ES}+\mathrm{APCI}) m / z: 316.0[\mathrm{M}+\mathrm{H}]^{+}$

(E)-2-(4-([1,1'-Biphenyl]-4-yl)but-3-en-1-yl)-4-methylbenzenesulfonamide. To a solution of (E)-2-(4([1,1'-biphenyl]-4-yl)but-3-en-1-yl)isoindoline-1,3-dione (300 $\mathrm{mg}, 0.9 \mathrm{mmol})$ in EtOH $(3.5 \mathrm{~mL})$ was added hydrazine monohydrate $(82 \mu \mathrm{L}, 1.7 \mathrm{mmol})$ according to General Procedure 2 to give $(E)-4-\left(\left[1,1^{\prime}-\right.\right.$ biphenyl]-4-yl)but-3-en-1-amine.

To a solution of $(E)-4-([1,1$ '-biphenyl $]-4-y l)$ but-3-en-1-amine $(189 \mathrm{mg}, 0.9 \mathrm{mmol})$ in $\mathrm{CH}_{2} \mathrm{Cl}_{2}(3.0 \mathrm{~mL})$ was added $\mathrm{Et}_{3} \mathrm{~N}(177 \mu \mathrm{L}, 1.3 \mathrm{mmol})$ followed by $p$-TsCl $(162 \mathrm{mg}, 0.9 \mathrm{mmol})$ and DMAP (31 mg, 0.3 
mmol) according to General Procedure 2. Purification by silica gel flash column chromatography (hexane:EtOAc 9:1) afforded the title compound ( $75 \mathrm{mg}, 0.2 \mathrm{mmol}, 23 \%$ ) as a white solid. m.p. $126-128^{\circ} \mathrm{C}$; IR (ATR) $/ \mathrm{cm}^{-1}: 3276,3051,3025,2922,2865 ;{ }^{1} \mathrm{H}$ NMR $\left(500 \mathrm{MHz}, \mathrm{CDCl}_{3}\right) \delta 7.76(\mathrm{~d}, J=8.2 \mathrm{~Hz}, 2 \mathrm{H})$, $7.59(\mathrm{~d}, J=7.3 \mathrm{~Hz}, 2 \mathrm{H}), 7.54(\mathrm{~d}, J=8.2 \mathrm{~Hz}, 2 \mathrm{H}), 7.44$ (at, $J=7.6 \mathrm{~Hz}, 2 \mathrm{H}), 7.36-7.34(\mathrm{~m}, 3 \mathrm{H}), 7.30(\mathrm{~d}$, $J=8.0 \mathrm{~Hz}, 2 \mathrm{H}), 6.40(\mathrm{~d}, J=15.9 \mathrm{~Hz}, 1 \mathrm{H}), 6.03(\mathrm{dt}, J=15.9,7.1 \mathrm{~Hz}, 1 \mathrm{H}), 4.59(\mathrm{t}, J=6.0 \mathrm{~Hz}, 1 \mathrm{H}), 3.14$ $3.10(\mathrm{~m}, 2 \mathrm{H}), 2.42(\mathrm{~s}, 3 \mathrm{H}), 2.41-2.37(\mathrm{~m}, 2 \mathrm{H}) ;{ }^{13} \mathrm{C} \mathrm{NMR}\left(126 \mathrm{MHz}, \mathrm{CDCl}_{3}\right) \delta 143.6,140.8,140.4,137.1$, 136.0, 132.9, 129.9, 128.9, 127.5, 127.4, 127.3, 127.0, 126.7, 125.8, 42.7, 33.2, 21.7; LRMS (ES + APCI) $m / z: 378.0\left[\mathrm{M}+\mathrm{NH}_{4}\right]^{+}$; HRMS (ESI-TOF) $m / z:[\mathrm{M}+\mathrm{H}]^{+}$calcd. for $\mathrm{C}_{23} \mathrm{H}_{24} \mathrm{NO}_{2} \mathrm{~S} 378.1528$; found 378.1522 . (E)-N-(4-(4-Chlorophenyl)but-3-en-1-yl)-4-methylbenzenesulfonamide. ${ }^{31}$ To a solution of (E)-2-(4-(4chlorophenyl)but-3-en-1-yl)isoindoline-1,3-dione (444 mg, $1.4 \mathrm{mmol})$ in EtOH $(5.5 \mathrm{~mL})$ was added hydrazine monohydrate (138 $\mu \mathrm{L}, 2.8 \mathrm{mmol})$ according to General Procedure 2 to give (E)-4-(4-chlorophenyl)but-3-en-1-amine.

To a solution of $(E)-4$-(4-chlorophenyl)but-3-en-1-amine (150 mg, $0.8 \mathrm{mmol})$ in $\mathrm{CH}_{2} \mathrm{Cl}_{2}(3.0 \mathrm{~mL})$ was added $\mathrm{Et}_{3} \mathrm{~N}(172 \mu \mathrm{L}, 1.2 \mathrm{mmol})$ followed by $p-\mathrm{TsCl}(157 \mathrm{mg}, 0.8 \mathrm{mmol})$ and DMAP (30 $\left.\mathrm{mg}, 0.3 \mathrm{mmol}\right)$ according to General Procedure 2. Purification by silica gel flash column chromatography (hexane:EtOAc 8:2) afforded the title compound ( $57 \mathrm{mg}, 0.2 \mathrm{mmol}, 20 \%$ ) as a white solid. m.p. $90-92{ }^{\circ} \mathrm{C}$; IR (ATR) $/ \mathrm{cm}^{-}$ 1: 3360, 3250, 2947, 2826; ${ }^{1} \mathrm{H}$ NMR (500 MHz, $\left.\mathrm{CDCl}_{3}\right) \delta 7.74(\mathrm{~d}, J=8.2 \mathrm{~Hz}, 2 \mathrm{H}), 7.27$ (d, $J=8.2 \mathrm{~Hz}$, 2H), $7.23(\mathrm{~d}, J=8.5 \mathrm{~Hz}, 2 \mathrm{H}), 7.18(\mathrm{~d}, J=8.5 \mathrm{~Hz}, 2 \mathrm{H}), 6.30(\mathrm{~d}, J=15.9 \mathrm{~Hz}, 1 \mathrm{H}), 5.96(\mathrm{dt}, J=15.9,7.0$ $\mathrm{Hz}, 1 \mathrm{H}), 4.78(\mathrm{t}, J=5.9 \mathrm{~Hz}, 1 \mathrm{H}), 3.10-3.07(\mathrm{~m}, 2 \mathrm{H}), 2.41(\mathrm{~s}, 3 \mathrm{H}), 2.37-2.33(\mathrm{~m}, 2 \mathrm{H}) ;{ }^{13} \mathrm{C} \mathrm{NMR}(126$ $\left.\mathrm{MHz}, \mathrm{CDCl}_{3}\right) \delta 143.6,137.1,135.5,133.1,132.0,129.9,128.8,127.5,127.2,126.6,42.6,33.1,21.6$; LRMS (ES + APCI) $m / z: 336.0[\mathrm{M}+\mathrm{H}]^{+}$.

(E)-N-(4-(3-Chlorophenyl)but-3-en-1-yl)-4-methylbenzenesulfonamide. To a solution of (E)-2-(4-(3-chlorophenyl)but-3-en-1-yl)isoindoline-1,3-dione (1.8 g, $5.8 \mathrm{mmol})$ in EtOH $(22 \mathrm{~mL})$ was added hydrazine monohydrate $(560 \mu \mathrm{L}, 11.5 \mathrm{mmol})$ according to General Procedure 2 to give $(E)$-4-(3-chlorophenyl)but3-en-1-amine. 
To a solution of (E)-4-(3-chlorophenyl)but-3-en-1-amine (1.1 g, $5.8 \mathrm{mmol})$ in $\mathrm{CH}_{2} \mathrm{Cl}_{2}(20 \mathrm{~mL})$ was added $\mathrm{Et}_{3} \mathrm{~N}(1.2 \mu \mathrm{L}, 8.7 \mathrm{mmol})$ followed by $p$ - TsCl $(1.1 \mathrm{~g}, 5.8 \mathrm{mmol})$ and DMAP $(0.2 \mathrm{~g}, 1.7 \mathrm{mmol})$ according to General Procedure 2. Purification by silica gel flash column chromatography (hexane:EtOAc 8:2) afforded the title compound ( $0.4 \mathrm{~g}, 1.2 \mathrm{mmol}, 21 \%$ ) as a colourless oil. IR (ATR)/ $\mathrm{cm}^{-1}: 3272,3060,3025$, 2921, 2870; ${ }^{1} \mathrm{H}$ NMR (500 MHz, $\left.\mathrm{CDCl}_{3}\right) \delta 7.74(\mathrm{~d}, J=8.0 \mathrm{~Hz}, 2 \mathrm{H}), 7.28(\mathrm{~d}, J=8.0 \mathrm{~Hz}, 2 \mathrm{H}), 7.24(\mathrm{~m}$, 1H), 7.21-7.17 (m, 2H), 7.13 (d, $J=7.1 \mathrm{~Hz}, 1 \mathrm{H}), 6.29$ (d, $J=15.9 \mathrm{~Hz}, 1 \mathrm{H}), 5.99$ (dt, $J=15.9,7.0 \mathrm{~Hz}$, 1H), $4.68(\mathrm{bs}, 1 \mathrm{H}), 3.12-3.08(\mathrm{~m}, 2 \mathrm{H}), 2.42(\mathrm{~s}, 3 \mathrm{H}), 2.39-2.35(\mathrm{~m}, 2 \mathrm{H}) ;{ }^{13} \mathrm{C} \mathrm{NMR}\left(126 \mathrm{MHz}, \mathrm{CDCl}_{3}\right) \delta$ 143.6, 138.8, 137.1, 134.6, 131.9, 129.9, 127.5, 127.4, 127.3, 126.1, 124.6, 42.6, 33.1, 21.7 (1 carbon missing); LRMS (ES + APCI) $m / z: 352.9\left[\mathrm{M}+\mathrm{NH}_{4}\right]^{+}$; HRMS (ESI-TOF) $m / z:[\mathrm{M}+\mathrm{H}]^{+}$calcd. for $\mathrm{C}_{17} \mathrm{H}_{19}{ }^{35} \mathrm{ClNO}_{2} \mathrm{~S} 336.0825$; found 336.0823 .

(E)-N-(4-(4-(1,3-Dioxalan-2-yl)phenyl)but-3-en-1-yl)-4-methylbenzenesulfonamide. To a solution of (E)2-(4-(4-(1,3-dioxalan-2-yl)phenyl)but-3-en-1-yl)isoindoline-1,3-dione (400 mg, $1.1 \mathrm{mmol})$ in EtOH (4.5 $\mathrm{mL})$ was added hydrazine monohydrate $(111 \mu \mathrm{L}, 2.3 \mathrm{mmol})$ according to General Procedure 2 to give $(E)$ (4-(4-(1,3-dioxalan-2-yl)phenyl)but-3-en-1-yl)-1-amine.

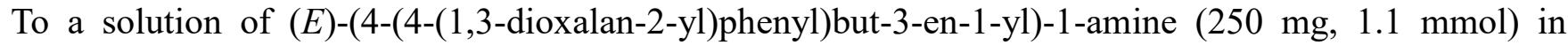
$\mathrm{CH}_{2} \mathrm{Cl}_{2}(4.0 \mathrm{~mL})$ was added $\mathrm{Et}_{3} \mathrm{~N}(240 \mu \mathrm{L}, 1.7 \mathrm{mmol})$ followed by $p$ - $\mathrm{TsCl}(217 \mathrm{mg}, 1.1 \mathrm{mmol})$ and DMAP (42 mg, $0.3 \mathrm{mmol}$ ) according to General Procedure 2. Purification by silica gel flash column chromatography (hexane:EtOAc 6:4) afforded the title compound (260 $\mathrm{mg}, 0.7 \mathrm{mmol}, 61 \%$ ) as a colourless oil. IR (ATR) $/ \mathrm{cm}^{-1}: 3264,3026,2948,2883 ;{ }^{1} \mathrm{H}$ NMR $\left(500 \mathrm{MHz}, \mathrm{CDCl}_{3}\right) \delta 7.74(\mathrm{~d}, J=8.3 \mathrm{~Hz}, 2 \mathrm{H}), 7.40(\mathrm{~d}, J$ $=8.2 \mathrm{~Hz}, 2 \mathrm{H}), 7.30-7.27(\mathrm{~m}, 4 \mathrm{H}), 6.36(\mathrm{~d}, J=15.9 \mathrm{~Hz}, 1 \mathrm{H}), 5.99(\mathrm{dt}, J=15.8,7.1 \mathrm{~Hz}, 1 \mathrm{H}), 5.79(\mathrm{~s}, 1 \mathrm{H})$, $4.43(\mathrm{t}, J=5.8 \mathrm{~Hz}, 1 \mathrm{H}), 4.15-4.11(\mathrm{~m}, 2 \mathrm{H}), 4.05-4.01(\mathrm{~m}, 2 \mathrm{H}), 3.13-3.08(\mathrm{~m}, 2 \mathrm{H}), 2.42(\mathrm{~s}, 3 \mathrm{H}), 2.39-$ $2.34(\mathrm{~m}, 2 \mathrm{H}) ;{ }^{13} \mathrm{C}$ NMR $\left(101 \mathrm{MHz}, \mathrm{CDCl}_{3}\right) \delta 143.6,137.8,137.3,137.1,133.0,130.0,127.3,126.9$, 126.33, 126.26, 103.7, 65.4, 42.6, 33.2, 21.7; LRMS (ES + APCI) m/z: $374.0[\mathrm{M}+\mathrm{H}]^{+}$; HRMS (ESI-TOF) $m / z:[\mathrm{M}+\mathrm{H}]^{+}$calcd. for $\mathrm{C}_{20} \mathrm{H}_{24} \mathrm{NO}_{4} \mathrm{~S} 374.1426$; found 374.1420. 
(E)-4-Methyl-N-(4-(4-trifluoromethyl)phenyl)but-3-en-1-yl)benzenesulfonamide. To a solution of (E)-2(4-(4-trifluoromethylphenyl)but-3-en-1-yl)isoindoline-1,3-dione (1.0 g, $2.9 \mathrm{mmol})$ in EtOH (11 mL) was added hydrazine monohydrate $(280 \mu \mathrm{L}, 5.8 \mathrm{mmol})$ according to General Procedure 2 to give $(E)-4-(4-$ (trifluoro)phenyl)but-3-en-1-amine.

To a solution of $(E)-4-\left(4-\left(\right.\right.$ trifluoro)phenyl)but-3-en-1-amine $(623 \mathrm{mg}, 3.0 \mathrm{mmol})$ in $\mathrm{CH}_{2} \mathrm{Cl}_{2}(9.6 \mathrm{~mL})$ was added $\mathrm{Et}_{3} \mathrm{~N}(604 \mu \mathrm{L}, 4.3 \mathrm{mmol})$ followed by $p$-TsCl $(551 \mathrm{mg}, 2.9 \mathrm{mmol})$ and DMAP (106 mg, $\left.0.9 \mathrm{mmol}\right)$ according to General Procedure 2. Purification by silica gel flash column chromatography (hexane:EtOAc 8:2) afforded the title compound (409 mg, $1.1 \mathrm{mmol}, 38 \%$ ) as a white solid. m.p. $121-122{ }^{\circ} \mathrm{C}$; IR (ATR) $/ \mathrm{cm}^{-1}: 3330,3244,2991,2875 ;{ }^{1} \mathrm{H}$ NMR $\left(500 \mathrm{MHz}, \mathrm{CDCl}_{3}\right) \delta 7.74(\mathrm{~d}, J=8.1 \mathrm{~Hz}, 2 \mathrm{H}), 7.55(\mathrm{~d}, J$ $=8.1 \mathrm{~Hz}, 2 \mathrm{H}), 7.37(\mathrm{~d}, J=8.0 \mathrm{~Hz}, 2 \mathrm{H}), 7.29(\mathrm{~d}, J=8.0 \mathrm{~Hz}, 2 \mathrm{H}), 6.40(\mathrm{~d}, J=15.9 \mathrm{~Hz}, 1 \mathrm{H}), 6.14-6.08$ $(\mathrm{m}, 1 \mathrm{H}), 4.39(\mathrm{t}, J=5.9 \mathrm{~Hz}, 1 \mathrm{H}), 3.15-3.11(\mathrm{~m}, 2 \mathrm{H}), 2.42(\mathrm{~s}, 3 \mathrm{H}), 2.42-2.39(\mathrm{~m}, 2 \mathrm{H}) ;{ }^{13} \mathrm{C}$ NMR $(126$ $\left.\mathrm{MHz} \mathrm{CDCl}_{3}\right) \delta 143.7,140.4,137.1,132.0,129.9,129.4\left(\mathrm{~d}, J_{\mathrm{C}-\mathrm{F}}=33.1 \mathrm{~Hz}\right), 128.7,127.3,126.4,125.6$ $\left(\mathrm{q}, J_{\mathrm{C}-\mathrm{F}}=3.7 \mathrm{~Hz}\right), 124.3\left(\mathrm{~d}, J_{\mathrm{C}-\mathrm{F}}=272.0 \mathrm{~Hz}\right), 42.6,33.3,21.6$; LRMS (ES + APCI) m/z: $370.0[\mathrm{M}+\mathrm{H}]^{+}$. N-(4,4-Diphenylbut-3-en-1-yl)-4-methylbenzenesulfonamide. To a solution of 2-(4,4-diphenylbut-3-en-1yl)isoindoline-1,3-dione $(280 \mathrm{mg}, 0.8 \mathrm{mmol})$ in $\mathrm{EtOH}(3.0 \mathrm{~mL})$ was added hydrazine monohydrate $(77$ $\mu \mathrm{L}, 1.6 \mathrm{mmol}$ ) according to General Procedure 2 to give 4,4-diphenylbut-3-en-1-amine.

To a solution of 4,4-diphenylbut-3-en-1-amine (176 mg, $0.8 \mathrm{mmol})$ in $\mathrm{CH}_{2} \mathrm{Cl}_{2}(2.6 \mathrm{~mL})$ was added $\mathrm{Et}_{3} \mathrm{~N}$ $(165 \mu \mathrm{L}, 1.2 \mathrm{mmol})$ followed by $p$-TsCl $(166 \mathrm{mg}, 0.9 \mathrm{mmol})$ and DMAP (29 $\mathrm{mg}, 0.2 \mathrm{mmol})$ according to General Procedure 2. Purification by silica gel flash column chromatography (hexane:EtOAc 8:2) afforded the title compound (200 mg, $0.5 \mathrm{mmol}, 67 \%$ ) as a colourless oil. IR (ATR)/ $\mathrm{cm}^{-1}: 3281,3053,3023$, 2922; ${ }^{1} \mathrm{H}$ NMR $\left(500 \mathrm{MHz}, \mathrm{CDCl}_{3}\right) \delta 7.72(\mathrm{~d}, J=8.3 \mathrm{~Hz}, 2 \mathrm{H}), 7.38-7.29(\mathrm{~m}, 3 \mathrm{H}), 7.27-7.22(\mathrm{~m}, 5 \mathrm{H})$, 7.20-7.15 (m, 2H), 7.12-7.10 (m, 2H), $5.97(\mathrm{t}, J=7.4 \mathrm{~Hz}, 1 \mathrm{H}), 4.76(\mathrm{bs}, 1 \mathrm{H}), 3.07-3.02(\mathrm{~m}, 2 \mathrm{H}), 2.41$ (s, 3H), 2.30-2.24 (m, 2H); ${ }^{13} \mathrm{C}$ NMR (101 MHz, $\left.\mathrm{CDCl}_{3}\right) \delta 144.7,143.4,142.1,139.6,137.0,129.8$, 128.4, 128.2, 127.35, 127.31, 127.2, 124.7, 43.1, 29.9, 21.6; LRMS (ES + APCI) m/z: $378.1[\mathrm{M}+\mathrm{H}]^{+}$; HRMS (ESI-TOF) $m / z$ : [M+H] $]^{+}$calcd. for $\mathrm{C}_{23} \mathrm{H}_{24} \mathrm{NO}_{2} \mathrm{~S} 378.1528$; found 378.1521. 
(E)-(4-phenylbut-3-en-1-yl)acetamide $(\boldsymbol{8 b}) .{ }^{32}$ To a cooled $\left(0^{\circ} \mathrm{C}\right)$ solution of $(E)$-4-phenylbut-3-en-1amine (200 mg, $1.4 \mathrm{mmol})$ in $\mathrm{CH}_{2} \mathrm{Cl}_{2}(6 \mathrm{~mL})$ was added $\mathrm{Et}_{3} \mathrm{~N}(206 \mu \mathrm{L}, 2.0 \mathrm{mmol})$ followed by addition of acetyl chloride ( $97 \mu \mathrm{L}, 1.4 \mathrm{mmol})$. The resultant mixture was then stirred at $\mathrm{rt}$ for $18 \mathrm{~h}$, before washing with a $1 \mathrm{M} \mathrm{HCl}$ solution $(20 \mathrm{~mL})$ followed by $1 \mathrm{M} \mathrm{NaOH}$ solution $(20 \mathrm{~mL})$ and brine $(20 \mathrm{~mL})$. The organic layer was dried over $\mathrm{MgSO}_{4}$, filtered and the solvent was removed by rotary evaporation affording the title compound $\mathbf{8 b}$ (190 mg, $1.0 \mathrm{mmol}, 72 \%$ ) as a yellow semi-solid. ${ }^{1} \mathrm{H} \mathrm{NMR}\left(400 \mathrm{MHz}, \mathrm{CDCl}_{3}\right) 7.35$ $7.28(\mathrm{~m}, 4 \mathrm{H}), 7.22(\mathrm{t}, J=7.1 \mathrm{~Hz}, 1 \mathrm{H}), 6.45(\mathrm{~d}, J=15.8 \mathrm{~Hz}, 1 \mathrm{H}), 6.14(\mathrm{dt}, J=15.8,7.1 \mathrm{~Hz}, 1 \mathrm{H}), 5.62(\mathrm{bs}$, 1H), 3.42-3.37 (m, 2H), 2.45-2.39 (m, 2H), $1.96(\mathrm{~s}, 3 \mathrm{H}) ;{ }^{13} \mathrm{C} \mathrm{NMR}\left(101 \mathrm{MHz}, \mathrm{CDCl}_{3}\right) \delta 170.2,137.3$, 132.5, 128.7, 127.5, 127.0, 126.2, 39.1, 33.2, 23.5; LRMS (ES + APCI) $m / z: 190.1[\mathrm{M}+\mathrm{H}]^{+}$.

tert-Butyl (E)-(4-phenylbut-3-en-1-yl)carbamate (8c) ${ }^{33}$ To a solution of (E)-4-phenylbut-3-en-1-amine (316 mg, $2.1 \mathrm{mmol})$ in $\mathrm{CH}_{2} \mathrm{Cl}_{2}(4 \mathrm{~mL})$ was added $\mathrm{K}_{2} \mathrm{CO}_{3}(591 \mathrm{mg}, 4.3 \mathrm{mmol})$ followed by addition of ditert-butyl dicarbonate $(468 \mathrm{mg}, 2.1 \mathrm{mmol})$. The resultant mixture was then stirred at $40{ }^{\circ} \mathrm{C}$ for $18 \mathrm{~h}$, before the addition of $\mathrm{H}_{2} \mathrm{O}(20 \mathrm{~mL})$ and the stirring was extended for an extra hour. Layers were separated and the organic layer was washed with brine $(30 \mathrm{~mL})$, dried over $\mathrm{MgSO}_{4}$, filtered and the solvent was removed by rotary evaporation. Purification by silica gel flash column chromatography (hexane:EtOAc 9:1) afforded the title compound $8 \mathrm{c}(441 \mathrm{mg}, 1.8 \mathrm{mmol}, 83 \%)$ as a colourless oil. ${ }^{1} \mathrm{H}$ NMR $\left(400 \mathrm{MHz}, \mathrm{CDCl}_{3}\right)$ $\delta 7.35-7.34$ (m, 2H), 7.30 (at, $J=7.5 \mathrm{~Hz}, 2 \mathrm{H}), 7.21$ (t, $J=7.1 \mathrm{~Hz}, 1 \mathrm{H}), 6.45$ (d, $J=15.8 \mathrm{~Hz}, 1 \mathrm{H}), 6.15$ (dt, $J=15.8,7.1 \mathrm{~Hz}, 1 \mathrm{H}), 4.59$ (bs, 1H), 3.30-3.25 (m, 2H), 2.43-2.38 (m, 2H), 1.44 (s, 9H); ${ }^{13} \mathrm{C}$ NMR $\left(101 \mathrm{MHz}, \mathrm{CDCl}_{3}\right) \delta 156.1,137.4,132.4,128.7,127.4,127.2,126.2,40.2,33.7,28.6$ (1 carbon missing); LRMS (ES + APCI) $m / z: 248.1[\mathrm{M}+\mathrm{H}]^{+}$.

Benzyl (E)-(4-phenylbut-3-en-1-yl)carbamate (8d). ${ }^{21}$ To a solution of (E)-4-phenylbut-3-en-1-amine (308 $\mathrm{mg}, 2.1 \mathrm{mmol})$ in $\mathrm{H}_{2} \mathrm{O}(10 \mathrm{~mL})$ and acetone $(21 \mathrm{~mL})$ was added $\mathrm{NaHCO}_{3}(200 \mathrm{mg}, 2.4 \mathrm{mmol})$ followed by addition of benzyl chloroformate $(335 \mu \mathrm{L}, 2.4 \mathrm{mmol})$. The resultant mixture was then stirred at $\mathrm{rt}$ for $18 \mathrm{~h}$, before evaporation of the solvent and the precipitate was filtered affording the title compound $\mathbf{8 d}$ (498 mg, $1.8 \mathrm{mmol}, 85 \%)$ as a white solid. m.p. $90-92{ }^{\circ} \mathrm{C} ;{ }^{1} \mathrm{H} \mathrm{NMR}\left(400 \mathrm{MHz}, \mathrm{CDCl}_{3}\right) \delta 7.35-7.28(\mathrm{~m}$, 
9H), 7.24-7.1 (m, 1H), $6.45(\mathrm{~d}, J=15.9 \mathrm{~Hz}, 1 \mathrm{H}), 6.14(\mathrm{dt}, J=15.9,7.3 \mathrm{~Hz}, 1 \mathrm{H}), 5.10(\mathrm{~s}, 2 \mathrm{H}), 4.83(\mathrm{bs}$, 1H), 3.38-3.34 (m, 2H), 2.46-2.41 (m, 2H); $\left.{ }^{13} \mathrm{C} \mathrm{NMR} \mathrm{(101} \mathrm{MHz,} \mathrm{CDCl}_{3}\right) \delta 156.3,137.1,136.6,132.5$, 128.6, 128.5, 128.5, 128.1, 127.3, 126.6, 126.1, 66.6, 40.5, 33.5; LRMS (ES + APCI) m/z: $282.1[\mathrm{M}+\mathrm{H}]^{+}$. (E)-2,4-Dinitro-N-(4-phenylbut-3-en-1-yl)benzenesulfonamide $(\boldsymbol{8} \boldsymbol{e})$. To a cooled $\left({ }^{\circ} \mathrm{C}\right)$ solution of $(E)-4-$ phenylbut-3-en-1-amine (200 mg, $1.4 \mathrm{mmol})$ in $\mathrm{CH}_{2} \mathrm{Cl}_{2}(14 \mathrm{~mL})$ was added $\mathrm{Et}_{3} \mathrm{~N}(284 \mu \mathrm{L}, 2.0 \mathrm{mmol})$ followed by addition of 2,4-dinitrobenzenesulfonyl chloride (471 mg, $1.8 \mathrm{mmol}$ ). The resultant mixture was then stirred at $\mathrm{rt}$ for $18 \mathrm{~h}$, before quenching with a saturated solution of $\mathrm{NH}_{4} \mathrm{Cl}(20 \mathrm{~mL})$. The organic layer was extracted with $\mathrm{CH}_{2} \mathrm{Cl}_{2}(3 \times 50 \mathrm{~mL})$, washed with brine $(100 \mathrm{~mL})$, dried over $\mathrm{MgSO}_{4}$, filtered and the solvent was removed by rotary evaporation. Purification by silica gel flash column chromatography (hexane:EtOAc 8:2) afforded the title compound $\mathbf{8 e}(502 \mathrm{mg}, 1.3 \mathrm{mmol}, 98 \%)$ as a yellow solid. m.p. ${ }^{130-132}{ }^{\circ} \mathrm{C} ;{ }^{1} \mathrm{H}$ NMR $\left(400 \mathrm{MHz}, \mathrm{CDCl}_{3}\right) \delta 8.37(\mathrm{~s}, 1 \mathrm{H}), 8.36-8.34(\mathrm{~m}, 1 \mathrm{H}), 8.27-8.24(\mathrm{~m}, 1 \mathrm{H})$, 7.24-7.15 (m, 3H), 7.11-7.08 (m, 2H), $6.29(\mathrm{~d}, J=15.8 \mathrm{~Hz}, 1 \mathrm{H}), 5.83(\mathrm{dt}, J=15.8,7.3 \mathrm{~Hz}, 1 \mathrm{H}), 5.41$ (bt, $J=5.6 \mathrm{~Hz}, 1 \mathrm{H}), 3.46-3.42(\mathrm{~m}, 2 \mathrm{H}), 2.46-2.41(\mathrm{~m}, 2 \mathrm{H}) ;{ }^{13} \mathrm{C} \mathrm{NMR}\left(101 \mathrm{MHz}, \mathrm{CDCl}_{3}\right) \delta 149.2,147.7$, $139.8,136.2,133.8,131.9,128.6,128.1,127.0,125.9,125.1,120.7,43.9,33.5 ;$ LRMS (ES + APCI) $m / z: 378.1$ $[\mathrm{M}+\mathrm{H}]^{+}$; HRMS (ESI-TOF) $m / z$ : $[\mathrm{M}+\mathrm{H}]^{+}$calcd. for $\mathrm{C}_{16} \mathrm{H}_{16} \mathrm{~N}_{3} \mathrm{O}_{6} \mathrm{~S} 378.0760$; found 378.0746.

General Procedure 3: Oxyamination procedure for the synthesis of pyrrolidines. Malonoyl peroxide 1 (1.5 equiv) was added to a solution of alkene 11 (1.0 equiv) in HFIP ( $0.5 \mathrm{M})$. The mixture was stirred at $\mathrm{rt}$ for $5 \mathrm{~h}$. The solvent was removed by rotary evaporation and the resulting residue was directly treated with $1 \mathrm{M} \mathrm{NaOH}$ :THF $(1: 1(0.1 \mathrm{M}))$. The solution was stirred at $60{ }^{\circ} \mathrm{C}$ for $18 \mathrm{~h}$, allowed to cool to $\mathrm{rt}$ and the aqueous phase was extracted with EtOAc $(\times 3)$. The combined organics were washed with brine and dried over $\mathrm{MgSO}_{4}$. Removal of the solvent under reduced pressure afforded the crude pyrrolidine product. Purification by silica gel flash column chromatography eluting with hexane:EtOAc mixtures afforded the target compound $\mathbf{1 2}$.

(土)-2-Phenyl-1-tosylpyrrolidin-3-ol (9a). ${ }^{34}$ Reaction of (E)-4-methyl-N-(4-phenylbut-3-en-1-yl)benzenesulfonamide 8a (25 mg, $0.08 \mathrm{mmol})$ and malonoyl peroxide 1 (16 mg, $0.12 \mathrm{mmol})$ in HFIP (0.2 $\mathrm{mL})$ 
according to General Procedure 3 followed by hydrolysis in $1 \mathrm{M} \mathrm{NaOH}$ :THF $(0.8 \mathrm{~mL}, 1: 1)$ gave the crude alcohol (1:13 cis:trans). Purification by silica gel flash column chromatography (hexane:EtOAc 4:6) gave the title compound $9 \mathrm{a}\left(18 \mathrm{mg}, 0.06 \mathrm{mmol}, 71 \%\right.$ ) as a white solid. m.p. $148-149{ }^{\circ} \mathrm{C}, \mathrm{Lit}^{34}\left[155-156{ }^{\circ} \mathrm{C}\right]$; IR (ATR) $/ \mathrm{cm}^{-1}:$ 3474, 3065, 3034, 2929, 2892; ${ }^{1} \mathrm{H}$ NMR $\left(500 \mathrm{MHz}, \mathrm{CDCl}_{3}\right) \delta 7.75(\mathrm{~d}, J=8.1 \mathrm{~Hz}, 2 \mathrm{H})$, 7.34-7.33 (m, 4H), $7.31(\mathrm{~d}, J=8.1 \mathrm{~Hz}, 2 \mathrm{H}), 7.28-7.26(\mathrm{~m}, 1 \mathrm{H}), 4.66(\mathrm{bs}, 1 \mathrm{H}), 4.18(\mathrm{bs}, 1 \mathrm{H}), 3.73(\mathrm{td}, J$ $=9.4,2.1 \mathrm{~Hz}, 1 \mathrm{H}), 3.53(\mathrm{td}, J=9.9,7.0 \mathrm{~Hz}, 1 \mathrm{H}), 2.43(\mathrm{~s}, 3 \mathrm{H}), 2.07-2.00(\mathrm{~m}, 1 \mathrm{H}), 1.77-1.73(\mathrm{~m}, 1 \mathrm{H})$, $1.37-1.38(\mathrm{~m}, 1 \mathrm{H}) ;{ }^{13} \mathrm{C} \mathrm{NMR}\left(126 \mathrm{MHz}, \mathrm{CDCl}_{3}\right) \delta 143.7,139.9,134.7,129.7,128.7,128.9,127.7,126.3$, 79.1, 72.0, 46.8, 31.5, 21.7; LRMS (ES + APCI) $m / z: 318.0[\mathrm{M}+\mathrm{H}]^{+}$.

(士)-2-(p-Tolyl)-1-tosylpyrrolidin-3-ol (13). Reaction of (E)-4-methyl- $N$-(4-(p-tolyl)but-3-en-1-yl)benzenesulfonamide (100 mg, $0.32 \mathrm{mmol})$ and malonoyl peroxide $1(61 \mathrm{mg}, 0.48 \mathrm{mmol})$ in $\mathrm{HFIP}(0.7 \mathrm{~mL})$ according to General Procedure 3 followed by hydrolysis in $1 \mathrm{M} \mathrm{NaOH}$ :THF $(3.2 \mathrm{~mL}, 1: 1)$ gave the crude alcohol (1:7 cis:trans). Purification by silica gel flash column chromatography (hexane:EtOAc 4:6) gave the title compound $13(69 \mathrm{mg}, 0.21 \mathrm{mmol}, 66 \%)$ as a white solid. m.p. $135-136{ }^{\circ} \mathrm{C}$; IR (ATR)/cm ${ }^{-1}: 3401$, 3029, 2960, 2899; ${ }^{1} \mathrm{H}$ NMR (400 MHz, $\left.\mathrm{CDCl}_{3}\right) \delta 7.73(\mathrm{~d}, J=8.2 \mathrm{~Hz}, 2 \mathrm{H}), 7.29(\mathrm{~d}, J=8.0 \mathrm{~Hz}, 2 \mathrm{H}), 7.22$ $(\mathrm{d}, J=8.2 \mathrm{~Hz}, 2 \mathrm{H}), 7.13(\mathrm{~d}, J=8.0 \mathrm{~Hz}, 2 \mathrm{H}), 4.62(\mathrm{bs}, 1 \mathrm{H}), 4.13(\mathrm{bs}, 1 \mathrm{H}), 3.70$ (ddd, $J=9.3,8.6,2.4 \mathrm{~Hz}$, $1 \mathrm{H}), 3.50(\mathrm{td}, J=9.9,6.9 \mathrm{~Hz}, 1 \mathrm{H}), 2.42(\mathrm{~s}, 3 \mathrm{H}), 2.33(\mathrm{~s}, 3 \mathrm{H}), 2.07-1.97(\mathrm{~m}, 1 \mathrm{H}), 1.75-1.69(\mathrm{~m}, 1 \mathrm{H}), 1.54$ (bs, $1 \mathrm{H}) ;{ }^{13} \mathrm{C}$ NMR $\left(101 \mathrm{MHz}, \mathrm{CDCl}_{3}\right) \delta 143.6,137.3,137.1,134.7,129.7,129.3,127.9,126.2,79.0$, 71.8, 46.8, 31.5, 21.7, 21.2; LRMS (ES + APCI) $m / z: 332.0[\mathrm{M}+\mathrm{H}]^{+}$; HRMS (ESI-TOF) $m / z:[\mathrm{M}+\mathrm{H}]^{+}$ calcd. for $\mathrm{C}_{18} \mathrm{H}_{22} \mathrm{NO}_{3} \mathrm{~S} 332.1320$; found 332.1316.

( \pm )-2-(m-Tolyl)-1-tosylpyrrolidin-3-ol (14). Reaction of (E)-4-methyl- $N$-(4-( $m$-tolyl)but-3-en-1-yl)benzenesulfonamide (50 mg, $0.16 \mathrm{mmol})$ and malonoyl peroxide 1 (30 mg, $0.24 \mathrm{mmol})$ in $\mathrm{HFIP}(0.3 \mathrm{~mL})$ according to General Procedure 3 followed by hydrolysis in $1 \mathrm{M} \mathrm{NaOH}$ :THF $(1.9 \mathrm{~mL}, 1: 1)$ gave the crude alcohol (1:6 cis:trans). Purification by silica gel flash column chromatography (hexane:EtOAc 4:6) gave

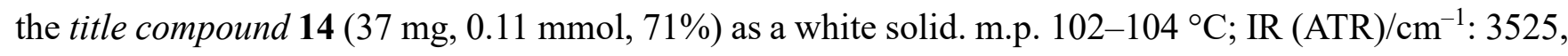
3489, 3478. 3462, 3447, 2950, 2921; ${ }^{1} \mathrm{H}$ NMR (400 MHz, $\left.\mathrm{CDCl}_{3}\right) \delta 7.73(\mathrm{~d}, J=8.3 \mathrm{~Hz}, 2 \mathrm{H}), 7.29(\mathrm{~d}, J$ 
$=8.3 \mathrm{~Hz}, 2 \mathrm{H}), 7.21($ at, $J=7.5 \mathrm{~Hz}, 1 \mathrm{H}), 7.12-7.10(\mathrm{~m}, 2 \mathrm{H}), 7.06(\mathrm{~d}, J=7.4 \mathrm{~Hz}, 1 \mathrm{H}), 4.63(\mathrm{bs}, 1 \mathrm{H}), 4.14$

(bs, 1H), 3.71 (ddd, $J=9.4,8.5,2.4 \mathrm{~Hz}, 1 \mathrm{H}), 3.53(\mathrm{td}, J=9.9,6.9 \mathrm{~Hz}, 1 \mathrm{H}), 2.42(\mathrm{~s}, 3 \mathrm{H}), 2.32(\mathrm{~s}, 3 \mathrm{H})$, 2.07-1.98 (m, 1H), 1.76-1.70 (m, 1H), $1.55(\mathrm{bd}, J=2.6 \mathrm{~Hz}, 1 \mathrm{H}) ;{ }^{13} \mathrm{C} \mathrm{NMR}\left(101 \mathrm{MHz}, \mathrm{CDCl}_{3}\right) \delta 143.6$, $139.9,138.3,134.8,129.7,128.6,128.4,127.8,127.0,123.4,79.0,72.0,46.9,31.5,21.7,21.6$; LRMS $(\mathrm{ES}+\mathrm{APCI}) \mathrm{m} / z: 332.0[\mathrm{M}+\mathrm{H}]^{+}$; HRMS (ESI-TOF) $m / z:[\mathrm{M}+\mathrm{H}]^{+}$calcd. for $\mathrm{C}_{18} \mathrm{H}_{22} \mathrm{NO}_{3} \mathrm{~S}$ 332.1320; found 332.1316 .

(土)-2-(o-Tolyl)-1-tosylpyrrolidin-3-ol (15). Reaction of (E)-4-methyl- $N$-(4-(o-tolyl)but-3-en-1-yl)benzenesulfonamide (100 mg, $0.32 \mathrm{mmol})$ and malonoyl peroxide $1(61 \mathrm{mg}, 0.48 \mathrm{mmol})$ in $\mathrm{HFIP}(0.6 \mathrm{~mL})$ according to General Procedure 3 followed by hydrolysis in $1 \mathrm{M} \mathrm{NaOH}$ :THF $(3.2 \mathrm{~mL}, 1: 1)$ gave the crude alcohol (1:4.6 cis:trans). Purification by silica gel flash column chromatography (hexane:EtOAc 4:6) gave the title compound 15 (76 mg, $0.23 \mathrm{mmol}, 72 \%$ ) as a white solid. m.p. $173-175{ }^{\circ} \mathrm{C}$; IR (ATR)/ $\mathrm{cm}^{-1}: 3504$, 3064, 2948, 2922, 2854; ${ }^{1} \mathrm{H}$ NMR (400 MHz, $\left.\mathrm{CDCl}_{3}\right) \delta 7.75(\mathrm{~d}, J=8.1 \mathrm{~Hz}, 2 \mathrm{H}), 7.36-7.34(\mathrm{~m}, 1 \mathrm{H}), 7.30$ $(\mathrm{d}, J=8.1 \mathrm{~Hz}, 2 \mathrm{H}), 7.20-7.12(\mathrm{~m}, 3 \mathrm{H}), 4.85(\mathrm{bs}, 1 \mathrm{H}), 4.06(\mathrm{bs}, 1 \mathrm{H}), 3.76(\mathrm{td}, J=8.9,1.6 \mathrm{~Hz}, 1 \mathrm{H}), 3.54$ (ddd, $J=11.0,9.3,6.7 \mathrm{~Hz}, 1 \mathrm{H}), 2.42(\mathrm{~s}, 3 \mathrm{H}), 2.38(\mathrm{~s}, 3 \mathrm{H}), 2.10-2.01(\mathrm{~m}, 1 \mathrm{H}), 1.79-1.74(\mathrm{~m}, 1 \mathrm{H}), 1.59$ (bs, $1 \mathrm{H}) ;{ }^{13} \mathrm{C} \mathrm{NMR}\left(150 \mathrm{MHz}, \mathrm{CDCl}_{3}\right) \delta 143.6,138.2,134.8,134.4,130.5,129.7,127.8,127.5,126.4$, 126.2, 77.6, 69.7, 46.9, 31.5, 21.7, 19.6; LRMS (ES + APCI) m/z: $332.0[\mathrm{M}+\mathrm{H}]^{+}$; HRMS (ESI-TOF) $m / z$ : $[\mathrm{M}+\mathrm{H}]^{+}$calcd. for $\mathrm{C}_{18} \mathrm{H}_{22} \mathrm{NO}_{3} \mathrm{~S} 332.1320$; found 332.1317 .

(土)-2-([1,1'-Biphenyl]-4-yl)-1-tosylpyrrolidin-3-ol (16). Reaction of (E)-2-(4-([1,1'-biphenyl]-4-yl)but-3en-1-yl)-4-methylbenzenesulfonamide (40 mg, $0.11 \mathrm{mmol})$ and malonoyl peroxide 1 (20 mg, $0.16 \mathrm{mmol})$ in HFIP $(0.2 \mathrm{~mL})$ according to General Procedure 3 followed by hydrolysis in $1 \mathrm{M} \mathrm{NaOH}: \mathrm{THF}(1.0 \mathrm{~mL}$, 1:1) gave the crude alcohol (1:9 cis:trans). Purification by silica gel flash column chromatography (hexane:EtOAc 4:6) gave the title compound $16(30 \mathrm{mg}, 0.08 \mathrm{mmol}, 72 \%)$ as a white solid. m.p. $190-192{ }^{\circ} \mathrm{C}$, decomp.; IR (ATR)/cm ${ }^{-1}: 3450,3010,2947,2920 ;{ }^{1} \mathrm{H}$ NMR $\left(500 \mathrm{MHz}, \mathrm{CDCl}_{3}\right) \delta 7.76(\mathrm{~d}, J=8.1 \mathrm{~Hz}$, 2H), 7.50-7.47 (m, 4H), 7.45-7.40 (m, 4H), 7.29-7.23 (m, 3H), $4.70(\mathrm{bs}, 1 \mathrm{H}), 4.22$ (bs, 1H), 3.77-3.73 (m, 1H), $3.55(\mathrm{dt}, J=16.8,8.5 \mathrm{~Hz}, 1 \mathrm{H}), 2.43(\mathrm{~s}, 3 \mathrm{H}), 2.11-2.05(\mathrm{~m}, 1 \mathrm{H}), 1.79-1.75(\mathrm{~m}, 1 \mathrm{H}), 1.50$ (bs, 
$1 \mathrm{H}) ;{ }^{13} \mathrm{C} \mathrm{NMR}\left(126 \mathrm{MHz}, \mathrm{CDCl}_{3}\right) \delta 143.7,140.9,140.7,139.0,134.7,129.7,128.9,127.9,127.5,127.3$, 126.8, 79.1, 71.8, 46.9, 31.6, 21.7 (missing 1 carbon); LRMS (ES + APCI) m/z: 394.0 [M+H] ; HRMS (ESI-TOF) $m / z:[\mathrm{M}+\mathrm{H}]^{+}$calcd. for $\mathrm{C}_{23} \mathrm{H}_{24} \mathrm{NO}_{3} \mathrm{~S}$ 394.1477; found 394.1472.

(土)-2-(4-Chlorophenyl)-1-tosylpyrrolidin-3-ol (17). Reaction of (E)-N-(4-(4-chlorophenyl)but-3-en-1yl)-4-methylbenzenesulfonamide $(100 \mathrm{mg}, 0.30 \mathrm{mmol})$ and malonoyl peroxide 1 (57 $\mathrm{mg}, 0.45 \mathrm{mmol})$ in HFIP (0.6 mL) according to General Procedure 3 followed by hydrolysis in $1 \mathrm{M} \mathrm{NaOH}: \mathrm{THF}(2.6 \mathrm{~mL}$, 1:1) gave the crude alcohol (1:9 cis:trans). Purification by silica gel flash column chromatography (hexane:EtOAc 4:6) gave the title compound $\mathbf{1 7}(69 \mathrm{mg}, 0.20 \mathrm{mmol}, 67 \%)$ as a white solid. m.p. $169-171{ }^{\circ} \mathrm{C}$; IR (ATR)/cm ${ }^{-1}: 3558,3499,2937,2889 ;{ }^{1} \mathrm{H}$ NMR $\left(500 \mathrm{MHz}, \mathrm{CDCl}_{3}\right) \delta 7.72(\mathrm{~d}, J=7.7 \mathrm{~Hz}, 2 \mathrm{H}), 7.31-$ $7.26(\mathrm{~m}, 6 \mathrm{H}), 4.60(\mathrm{bs}, 1 \mathrm{H}), 4.11(\mathrm{bs}, 1 \mathrm{H}), 3.72-3.69(\mathrm{~m}, 1 \mathrm{H}), 3.52-3.47(\mathrm{~m}, 1 \mathrm{H}), 2.42(\mathrm{~s}, 3 \mathrm{H}), 2.02-1.95$ $(\mathrm{m}, 1 \mathrm{H}), 1.75-1.72(\mathrm{~m}, 1 \mathrm{H}), 1.58(\mathrm{bs}, 1 \mathrm{H}) ;{ }^{13} \mathrm{C} \mathrm{NMR}\left(126 \mathrm{MHz}, \mathrm{CDCl}_{3}\right) \delta 143.8,138.6,134.4,129.8$, 128.8, 128.9, 127.9, 127.7, 78.9, 71.4, 46.9, 31.5, 21.7; LRMS (ES + APCI) m/z: 351.9 [M] $]^{+}$HRMS (ESI-TOF) $m / z$ : $[\mathrm{M}+\mathrm{H}]^{+}$calcd. for $\mathrm{C}_{17} \mathrm{H}_{19}{ }^{35} \mathrm{ClNO}_{3} \mathrm{~S}$ 352.0774; found 352.0772.

(土)-2-(3-Chlorophenyl)-1-tosylpyrrolidin-3-ol (18). Reaction of (E)-N-(4-(3-chlorophenyl)but-3-en-1yl)-4-methylbenzenesulfonamide $(60 \mathrm{mg}, 0.18 \mathrm{mmol})$ and malonoyl peroxide 1 (34 $\mathrm{mg}, 0.27 \mathrm{mmol})$ in HFIP (0.4 mL) according to General Procedure 3 followed by hydrolysis in $1 \mathrm{M} \mathrm{NaOH}: \mathrm{THF}(0.4 \mathrm{~mL}$, 1:1) gave the crude alcohol (1:6 cis:trans). Purification by silica gel flash column chromatography (hexane:EtOAc 4:6) gave the title compound $\mathbf{1 8}(33 \mathrm{mg}, 0.09 \mathrm{mmol}, 52 \%)$ as a white solid. m.p. $96-98^{\circ} \mathrm{C}$; IR (ATR) $/ \mathrm{cm}^{-1}:$ 3489, 3062, 2952, 2922, 2954; ${ }^{1} \mathrm{H}$ NMR (500 MHz, $\left.\mathrm{CDCl}_{3}\right) \delta 7.72(\mathrm{~d}, J=8.1 \mathrm{~Hz}, 2 \mathrm{H}), 7.29$ $(\mathrm{d}, J=8.1 \mathrm{~Hz}, 2 \mathrm{H}), 7.27(\mathrm{~s}, 1 \mathrm{H}), 7.25-7.21(\mathrm{~m}, 3 \mathrm{H}), 4.63(\mathrm{bs}, 1 \mathrm{H}), 4.12(\mathrm{bs}, 1 \mathrm{H}), 3.70(\mathrm{td}, J=9.3,2.3$ $\mathrm{Hz}, 1 \mathrm{H}), 3.52(\mathrm{td}, J=9.9,6.9 \mathrm{~Hz}, 1 \mathrm{H}), 2.42(\mathrm{~s}, 3 \mathrm{H}), 2.06-1.96(\mathrm{~m}, 1 \mathrm{H}), 1.77-1.73(\mathrm{~m}, 2 \mathrm{H}) ;{ }^{13} \mathrm{C} \mathrm{NMR}$ $\left(126 \mathrm{MHz} \mathrm{CDCl}_{3}\right) \delta 143.9,142.2,134.6,134.5,130.0,129.9,129.8,127.8,126.5,124.6,78.9,71.4,46.9$, 31.6, 21.7; LRMS (ES + APCI) $m / z: 351.9[\mathrm{M}]^{+}$; HRMS (ESI-TOF) $m / z:[\mathrm{M}+\mathrm{H}]^{+}$calcd. for $\mathrm{C}_{17} \mathrm{H}_{19}{ }^{35} \mathrm{ClNO}_{3} \mathrm{~S} 352.0774$; found 352.0770. 
( \pm -2-(4-(1,3-Dioxalan-2-yl)phenyl)-1-tosylpyrrolidin-3-ol (19). Reaction of (E)-N-(4-(4-(1,3-dioxalan2-yl)phenyl)but-3-en-1-yl)-4-methylbenzenesulfonamide (200 mg, $0.54 \mathrm{mmol}$ ) and malonoyl peroxide 1 (103 mg, $0.80 \mathrm{mmol})$ in HFIP $(1.1 \mathrm{~mL})$ according to General Procedure 3 followed by hydrolysis in $1 \mathrm{M}$ NaOH:THF (5.3 mL, 1:1) gave the crude alcohol (1:9 cis:trans). Purification by silica gel flash column chromatography (hexane:EtOAc 1:1) gave the title compound $19(115 \mathrm{mg}, 0.30 \mathrm{mmol}, 55 \%)$ as a white solid. m.p. $146-148{ }^{\circ} \mathrm{C}$; IR (ATR)/(cm ${ }^{-1}: 3517,3054,2922,2887,2852,1702 ;{ }^{1} \mathrm{H}$ NMR $\left(400 \mathrm{MHz}, \mathrm{CDCl}_{3}\right)$ $\delta 7.72(\mathrm{~d}, J=8.2 \mathrm{~Hz}, 2 \mathrm{H}), 7.43(\mathrm{~d}, J=8.2 \mathrm{~Hz}, 2 \mathrm{H}), 7.34(\mathrm{~d}, J=8.0 \mathrm{~Hz}, 2 \mathrm{H}), 7.28(\mathrm{~d}, J=8.0 \mathrm{~Hz}, 2 \mathrm{H})$, $5.79(\mathrm{~s}, 1 \mathrm{H}), 4.66(\mathrm{bs}, 1 \mathrm{H}), 4.13-4.07(\mathrm{~m}, 3 \mathrm{H}), 4.05-4.00(\mathrm{~m}, 2 \mathrm{H}), 3.71-3.66(\mathrm{~m}, 1 \mathrm{H}), 3.51(\mathrm{dt}, J=9.9$, $6.9 \mathrm{~Hz}, 1 \mathrm{H}), 2.41(\mathrm{~s}, 3 \mathrm{H}), 2.01-1.92(\mathrm{~m}, 1 \mathrm{H}), 1.73-1.75(\mathrm{~m}, 2 \mathrm{H}) ;{ }^{13} \mathrm{C} \mathrm{NMR}\left(101 \mathrm{MHz}, \mathrm{CDCl}_{3}\right) \delta 143.7$, 141.1, 137.4, 134.7, 129.7, 127.9, 126.8, 126.4, 103.6, 78.9, 71.8, 65.4, 46.9, 31.4, 21.7; LRMS (ES + APCI) $m / z: 390.0[\mathrm{M}+\mathrm{H}]^{+}$; HRMS (ESI-TOF) $m / z:[\mathrm{M}+\mathrm{H}]^{+}$calcd. for $\mathrm{C}_{20} \mathrm{H}_{24} \mathrm{NO}_{5} \mathrm{~S}$ 390.1375; found 390.1370.

( \pm -1-Tosyl-2-(4-(trifluoromethyl)phenyl)pyrrolidine-3-ol (20). Reaction of (E)-4-methyl- $N$-(4-(4-trifluoromethyl)phenyl)but-3-en-1-yl)benzenesulfonamide (50 mg, $0.1 \mathrm{mmol})$ and malonoyl peroxide 1 ( $35 \mathrm{mg}$, $0.3 \mathrm{mmol})$ in HFIP $(0.6 \mathrm{~mL})$ according to General Procedure 3 warming up to $50^{\circ} \mathrm{C}$, followed by hydrolysis in $1 \mathrm{M} \mathrm{NaOH}$ :THF $(2.6 \mathrm{~mL}, 1: 1)$ gave the crude alcohol. Purification by silica gel flash column chromatography (hexane:EtOAc 4:6) gave the title compound $\mathbf{2 0}(10 \mathrm{mg}, 0.03 \mathrm{mmol}, 19 \%)$ as a white solid. m.p. $119-120{ }^{\circ} \mathrm{C}$; IR (ATR)/ $\mathrm{cm}^{-1}: 3541,2996,2888 ;{ }^{1} \mathrm{H}$ NMR $\left(500 \mathrm{MHz}, \mathrm{CDCl}_{3}\right) \delta 7.72(\mathrm{~d}, J=$ $8.0 \mathrm{~Hz}, 2 \mathrm{H}), 7.57$ (d, $J=8.0 \mathrm{~Hz}, 2 \mathrm{H}), 7.46$ (d, $J=8.0 \mathrm{~Hz}, 2 \mathrm{H}), 7.30$ (d, $J=8.0 \mathrm{~Hz}, 2 \mathrm{H}), 4.69$ (bs, 1H), $4.15(\mathrm{bs}, 1 \mathrm{H}), 3.74-3.70(\mathrm{~m}, 1 \mathrm{H}), 3.55-3.50(\mathrm{~m}, 1 \mathrm{H}), 2.42(\mathrm{~s}, 3 \mathrm{H}), 2.01-1.95(\mathrm{~m}, 1 \mathrm{H}), 1.78-1.74(\mathrm{~m}, 2 \mathrm{H})$; ${ }^{13} \mathrm{C}$ NMR $\left(126 \mathrm{MHz}, \mathrm{CDCl}_{3}\right) \delta 144.1,144.0,134.3,130.0\left(J_{\mathrm{C}-\mathrm{F}}=13.8 \mathrm{~Hz}\right), 129.8,127.9,126.8,125.7$ $\left(J_{\mathrm{C}-\mathrm{F}}=3.6 \mathrm{~Hz}\right), 124.2\left(J_{\mathrm{C}-\mathrm{F}}=272.0 \mathrm{~Hz}\right), 78.9,71.5,47.0,31.6,21.7$; LRMS (ES + APCI) $m / z: 386.0$ $[\mathrm{M}+\mathrm{H}]^{+}$; HRMS (ESI-TOF) $m / z$ : $[\mathrm{M}+\mathrm{H}]^{+}$calcd. for $\mathrm{C}_{18} \mathrm{H}_{19} \mathrm{~F}_{3} \mathrm{NO}_{3} \mathrm{~S}$ 386.1038; found 386.1030 .

(土)-2,2-Diphenyl-1-tosylpyrrolidin-3-ol (21). Reaction of N-(4,4-diphenylbut-3-en-1-yl)-4-methylbenzenesulfonamide (80 mg, $0.21 \mathrm{mmol})$ and malonoyl peroxide $1(41 \mathrm{mg}, 0.32 \mathrm{mmol})$ in HFIP $(0.4 \mathrm{~mL})$ 
according to General Procedure 3 followed by hydrolysis in $1 \mathrm{M} \mathrm{NaOH}$ :THF $(2.1 \mathrm{~mL}, 1: 1)$ gave the crude alcohol. Purification by silica gel flash column chromatography (hexane:EtOAc 6:4) gave the title compound 21 (68 mg, $0.17 \mathrm{mmol}, 82 \%$ ) as a white solid. m.p. $153-154{ }^{\circ} \mathrm{C}$; IR (ATR)/cm ${ }^{-1}: 3502,3054,2980$, 2954; ${ }^{1} \mathrm{H}$ NMR (400 MHz, $\left.\mathrm{CDCl}_{3}\right) \delta$ 7.49-7.47 (m, 2H), 7.43-7.34 (m, 3H), 7.30-7.25 (m, 3H), 7.20$7.17(\mathrm{~m}, 2 \mathrm{H}), 6.96(\mathrm{~d}, J=8.1 \mathrm{~Hz}, 2 \mathrm{H}), 6.86-6.83(\mathrm{~m}, 2 \mathrm{H}), 4.79(\mathrm{dd}, J=7.9,6.0 \mathrm{~Hz}, 1 \mathrm{H}), 4.04(\mathrm{ddd}, J=$ 9.4, 8.4, 3.9 Hz, 1H), 3.62 (ddd, $J=9.4,8.4,7.2 \mathrm{~Hz}, 1 \mathrm{H}), 2.33(\mathrm{~s}, 3 \mathrm{H}), 2.17-2.10(\mathrm{~m}, 1 \mathrm{H}), 1.77-1.67(\mathrm{~m}$, 1H), $1.43(\mathrm{bs}, 1 \mathrm{H}) ;{ }^{13} \mathrm{C} \mathrm{NMR}\left(101 \mathrm{MHz}, \mathrm{CDCl}_{3}\right) \delta 142.3,139.0,138.5,138.2,130.8,130.0,128.9,127.93$ 127.89, 127.7, 126.6, 79.2, 77.1, 46.4, 30.6, 21.5 (1 carbon missing); LRMS (ES + APCI) m/z: 394.0 $[\mathrm{M}+\mathrm{H}]^{+}$; HRMS (ESI-TOF) $m / z:[\mathrm{M}+\mathrm{H}]^{+}$calcd. for $\mathrm{C}_{23} \mathrm{H}_{24} \mathrm{NO}_{3} \mathrm{~S}$ 394.1477; found 394.1471.

(士)-2-Phenyl-1-tosylpyrrolidin-3-ol (22). Malonoyl peroxide 1 (45 $\mathrm{mg}, 0.35 \mathrm{mmol}$ ) was added to a solution of (Z)-4-methyl- $N$-(4-(p-tolyl)but-3-en-1-yl)benzenesulfonamide ${ }^{35}$ (70 mg, 0.23 mmol) in HFIP (0.5 $\mathrm{mL}$ ) and mixture stirred at $\mathrm{rt}$ for $5 \mathrm{~h}$. The solvent was removed by rotary evaporation and the resulting residue was directly treated with $1 \mathrm{M} \mathrm{NaOH}$ :THF $(2 \mathrm{~mL}(1: 1))$. The solution was stirred at $60{ }^{\circ} \mathrm{C}$ for 18 h, allowed to cool to $\mathrm{rt}$ and the aqueous phase was extracted with EtOAc $(3 \times 50 \mathrm{~mL})$. The combined organics were washed with brine $(100 \mathrm{~mL})$ and dried over $\mathrm{MgSO}_{4}$. Removal of the solvent under reduced pressure afforded the crude pyrrolidine product (4:1 cis:trans). Purification by silica gel flash column chromatography (EtOAc:Hexane 4:6) gave the title compound $22(37 \mathrm{mg}, 0.11 \mathrm{mmol}, 50 \%)$, as a white solid. m.p. $114-116{ }^{\circ} \mathrm{C}$; IR (ATR)/ $\mathrm{cm}^{-1}: 3452,2974,2872,1325,1156 ;{ }^{1} \mathrm{H}$ NMR (400 MHz, $\left.\mathrm{CDCl}_{3}\right) \delta$ 7.69-7.64 (m, 2H), 7.39-7.28 (m, 7H), $4.74(\mathrm{~d}, J=5.6 \mathrm{~Hz}, 1 \mathrm{H}), 4.25-4.15(\mathrm{~m}, 1 \mathrm{H}), 3.80-3.70(\mathrm{~m}, 1 \mathrm{H})$, $3.64(\mathrm{ddd}, J=10.7,7.7,4.9 \mathrm{~Hz}, 1 \mathrm{H}), 2.43(\mathrm{~s}, 3 \mathrm{H}), 1.89(\mathrm{ddt}, J=16.3,6.5,4.9 \mathrm{~Hz}, 1 \mathrm{H}), 1.80-1.69$ (m, 1H), $1.09(\mathrm{~d}, \mathrm{~J}=4.9 \mathrm{~Hz}, 1 \mathrm{H}) ;{ }^{13} \mathrm{C} \mathrm{NMR}\left(101 \mathrm{MHz}, \mathrm{CDCl}_{3}\right) \delta 143.7,136.5,135.0,129.8,128.8,128.7$, $128.3,127.9,127.7,126.3,73.7,67.7,47.2,32.5,21.7$, LRMS (ES + APCI) $m / z: 318.0[\mathrm{M}+\mathrm{H}]^{+}$.

2-(3-Bromo-4,4-diphenylbut-3-en-1-yl)isoindoline-1,3-dione. Bromine (0.3 mL, $5.7 \mathrm{mmol})$ was added dropwise to a solution of 2-(4,4-diphenylbut-3-en-1-yl)isoindoline-1,3-dione (1.0 g, $2.8 \mathrm{mmol})$ in 1,2DCE $(5 \mathrm{~mL})$ at room temperature. The resulting solution was stirred for $15 \mathrm{~h}$ before the solvent was 
removed under reduced pressure. The residue was dissolved with a solution of $\mathrm{KOH}(0.6 \mathrm{~g}, 11.3 \mathrm{mmol})$ in $\mathrm{MeOH}(10 \mathrm{~mL})$ and stirred for $30 \mathrm{~min}$. The solids were then separated by filtration and filtrate was concentrated under reduced pressure affording the title compound $(1.2 \mathrm{~g}, 2.7 \mathrm{mmol}, 98 \%)$ as a cream solid that was used without further purification. m.p. $192-193^{\circ} \mathrm{C}$; IR (ATR)/cm ${ }^{-1}: 2942,1699,1123,695 ;{ }^{1} \mathrm{H}$ $\operatorname{NMR}\left(400 \mathrm{MHz}, \mathrm{CDCl}_{3}\right) \delta$ 7.88-7.81 (m, 2H), 7.78-7.71 (m, 2H), 7.38-7.24 (m, 5H), 7.23-7.17 (m, 1H), 7.16-7.09 (m, 2H), 7.01-6.95 (m, 2H), $4.02(\mathrm{t}, J=6.3 \mathrm{~Hz}, 2 \mathrm{H}), 3.08(\mathrm{t}, J=6.3 \mathrm{~Hz}, 2 \mathrm{H}) ;{ }^{13} \mathrm{C}$ NMR $(101$ $\left.\mathrm{MHz}, \mathrm{CDCl}_{3}\right) \delta 168.0,144.4,142.9,139.8,133.9,132.3,129.0,128.5,128.6,128.1,127.4,127.4,123.3$, 122.9, 37.7, 37.0; LCMS (ES + APCI) $m / z: 331.9,434.0[\mathrm{M}+\mathrm{H}]^{+}$; HRMS (ESI-TOF) $m / z:[\mathrm{M}+\mathrm{H}]^{+}$calcd. for $\mathrm{C}_{24} \mathrm{H}_{19}{ }^{79} \mathrm{BrNO}_{2} 432.0599$; found 432.0589 .

2-(3-(Diphenylmethylene)pent-4-en-1-yl)isoindoline-1,3-dione. To a solution of 2-(3-bromo-4,4-diphenylbut-3-en-1-yl)isoindoline-1,3-dione $(1.0 \mathrm{~g}, 2.3 \mathrm{mmol})$ in degassed EtOH $(10 \mathrm{~mL})$ was added potassium vinyltrifluoroborate $(0.4 \mathrm{~g}, 2.8 \mathrm{mmol})$, and $\mathrm{Pd}(\mathrm{dppf}) \mathrm{Cl}_{2}(0.2 \mathrm{~g}, 0.2 \mathrm{mmol})$ under a $\mathrm{N}_{2}$ atmosphere. The resulting mixture was stirred at $\mathrm{rt}$ before the addition of $\mathrm{Et}_{3} \mathrm{~N}(0.8 \mathrm{~mL}, 5.6 \mathrm{mmol})$. The mixture was then heated to $120{ }^{\circ} \mathrm{C}$ and stirring was continued for $48 \mathrm{~h}$. The reaction was allowed to cool to room temperature and the solvent removed under reduced pressure. The resulting solid was dissolved in $\mathrm{CH}_{2} \mathrm{Cl}_{2}(20$ $\mathrm{mL})$, washed with $\mathrm{H}_{2} \mathrm{O}(20 \mathrm{~mL})$, dried over $\mathrm{MgSO}_{4}$, filtered and the solvent was removed by rotary evaporation. Purification by silica gel flash column chromatography (petroleum ether $\left(40-60{ }^{\circ} \mathrm{C}\right):$ EtOAc $\left.9: 1\right)$ afforded the title compound (350 $\mathrm{mg}, 0.9 \mathrm{mmol}, 40 \%(80 \%$ pure $)$ ) as a cream solid. m.p. $135-137^{\circ} \mathrm{C}$; IR $(\mathrm{ATR}) / \mathrm{cm}^{-1}: 3024,1696,1399,1104,988 ;{ }^{1} \mathrm{H} \mathrm{NMR}\left(400 \mathrm{MHz}, \mathrm{CDCl}_{3}\right) \delta 7.80(\mathrm{dd}, J=5.5,3.0 \mathrm{~Hz}, 2 \mathrm{H})$, $7.70(\mathrm{dd}, J=5.5,3.0 \mathrm{~Hz}, 2 \mathrm{H}), 7.37-7.14(\mathrm{~m}, 8 \mathrm{H}), 7.09(\mathrm{dd}, J=6.5,3.1 \mathrm{~Hz}, 2 \mathrm{H}), 6.58(\mathrm{dd}, J=17.5,11.0$ $\mathrm{Hz}, 1 \mathrm{H}), 5.61(\mathrm{~d}, J=17.5 \mathrm{~Hz}, 1 \mathrm{H}), 5.19(\mathrm{~d}, J=11.0 \mathrm{~Hz}, 1 \mathrm{H}), 3.89(\mathrm{t}, J=7.2 \mathrm{~Hz}, 2 \mathrm{H}), 2.82(\mathrm{t}, J=7.2$ $\mathrm{Hz}, 2 \mathrm{H}) ;{ }^{13} \mathrm{C} \mathrm{NMR}\left(101 \mathrm{MHz}, \mathrm{CDCl}_{3}\right) \delta 168.1,144.4,142.2,141.9,136.0,133.7,132.3,130.2,129.1$, $128.9,128.5,128.2,127.9,126.9,123.1,114.5,37.5,28.1 ;$ LCMS $(\mathrm{ES}+\mathrm{APCI}) \mathrm{m} / z:=380.5[\mathrm{M}+\mathrm{H}]^{+}$; HRMS (ESI-TOF) $m / z$ : [M+H $]^{+}$calcd. for $\mathrm{C}_{26} \mathrm{H}_{22} \mathrm{NO}_{2} 380.1651$; found 380.1638 . 
2-(3-(Diphenylmethylene)-5-hydroxypentyl)isoindoline-1,3-dione. To a solution of 2-(3-(diphenylmethylene)pent-4-en-1-yl)isoindoline-1,3-dione (220 mg, $0.6 \mathrm{mmol})$ in anhydrous THF (2 mL) was added $\mathrm{BH}_{3}(1.0 \mathrm{M}$ in THF, $0.9 \mathrm{~mL}, 0.9 \mathrm{mmol})$ under a $\mathrm{N}_{2}$ atmosphere. The resulting solution was stirred at $\mathrm{rt}$ for $4 \mathrm{~h}$. After this time a $2 \mathrm{M}$ solution of $\mathrm{NaOH}(2 \mathrm{~mL})$ was added followed by $\mathrm{H}_{2} \mathrm{O}_{2}(28 \% w / w, 2 \mathrm{~mL})$ and the mixture was further stirred for $2 \mathrm{~h}$. Upon completion, the reaction was quenched by the addition of a $2 \mathrm{M}$ solution of $\mathrm{HCl}(10 \mathrm{~mL})$ and the organic layer was extracted with $\mathrm{CH}_{2} \mathrm{Cl}_{2}(2 \times 10 \mathrm{~mL})$. The combined organics were washed with a saturated solution of $\mathrm{Na}_{2} \mathrm{~S}_{2} \mathrm{O}_{5}(2 \times 10 \mathrm{~mL})$, dried over $\mathrm{MgSO}_{4}$, filtered and the solvent was removed by rotary evaporation. Purification by silica gel flash column chromatography (petroleum ether $\left(40-60^{\circ} \mathrm{C}\right)$ :EtOAc $\left.1: 1\right)$ afforded the title compound $(80 \mathrm{mg}, 0.2 \mathrm{mmol}, 33 \%)$ as a cream solid. m.p. $137-139^{\circ} \mathrm{C}$; IR (ATR)/(cm ${ }^{-1}: 3434,2964,1685,1399,1043 ;{ }^{1} \mathrm{H}$ NMR (400 MHz, $\left.\mathrm{CDCl}_{3}\right) \delta$ $7.79(\mathrm{dd}, J=5.4,3.2 \mathrm{~Hz}, 2 \mathrm{H}), 7.70(\mathrm{dd}, J=5.4,3.2 \mathrm{~Hz}, 2 \mathrm{H}), 7.31-7.24(\mathrm{~m}, 2 \mathrm{H}), 7.21-7.07(\mathrm{~m}, 4 \mathrm{H})$, 7.06-7.00 (m, 2H), 6.87-6.83 (m, 2H), $3.81(\mathrm{t}, J=6.5 \mathrm{~Hz}, 2 \mathrm{H}), 3.75(\mathrm{t}, J=6.7 \mathrm{~Hz}, 2 \mathrm{H}), 2.68(\mathrm{t}, J=6.5$ $\mathrm{Hz}, 2 \mathrm{H}), 2.62(\mathrm{t}, J=6.7 \mathrm{~Hz}, 2 \mathrm{H}), 1.77(\mathrm{bs}, 1 \mathrm{H}) ;{ }^{13} \mathrm{C} \mathrm{NMR}\left(101 \mathrm{MHz}, \mathrm{CDCl}_{3}\right) \delta 168.1,143.7,142.6$, $142.1,133.8,132.4,131.5,129.0,128.9,128.2,128.1,126.5,126.4,123.1,61.3,36.4,35.7,30.6$; LCMS $(\mathrm{ES}+\mathrm{APCI}) m / z:=398.3[\mathrm{M}+\mathrm{H}]^{+}$; HRMS (ESI-TOF) $m / z:[\mathrm{M}+\mathrm{H}]^{+}$calcd. for $\mathrm{C}_{26} \mathrm{H}_{24} \mathrm{NO}_{3}$ 398.1756; found 398.1755 .

N-(3-(Diphenylmethylene)-5-hydroxypentyl)-4-methylbenzenesulfonamide (28). To a solution of 2-(3-(diphenylmethylene)-5-hydroxypentyl)isoindoline-1,3-dione $(120 \mathrm{mg}, 0.3 \mathrm{mmol})$ in EtOH $(2 \mathrm{~mL})$ was added hydrazine monohydrate $(30 \mu \mathrm{L}, 0.6 \mathrm{mmol})$ according to General Procedure 2 to give 5-amino-3(diphenylmethylene)pentan-1-ol.

To a solution of 5-amino-3-(diphenylmethylene)pentan-1-ol in $\mathrm{CH}_{2} \mathrm{Cl}_{2}(2.0 \mathrm{~mL})$ was added $\mathrm{Et} 3 \mathrm{~N}(60 \mu \mathrm{L}$, $0.3 \mathrm{mmol})$ followed by $p$-TsCl $(58 \mathrm{mg}, 0.3 \mathrm{mmol})$ and DMAP (11 $\mathrm{mg}, 0.09 \mathrm{mmol})$ according to General Procedure 2. Purification by silica gel flash column chromatography (cyclohexane:EtOAc 7:3) afforded the title compound 28 (67 mg, $0.2 \mathrm{mmol}, 53 \%$ ) as a colourless gum. IR (ATR)/cm ${ }^{-1}: 2878,1322,1153$, 700; ${ }^{1} \mathrm{H} \mathrm{NMR}\left(600 \mathrm{MHz}, \mathrm{CDCl}_{3}\right) \delta 7.66(\mathrm{~d}, J=8.1 \mathrm{~Hz}, 2 \mathrm{H}), 7.29-7.23(\mathrm{~m}, 6 \mathrm{H}), 7.21-7.17(\mathrm{~m}, 2 \mathrm{H}), 7.16-$ 
$7.12(\mathrm{~m}, 2 \mathrm{H}), 7.10-7.06(\mathrm{~m}, 2 \mathrm{H}), 4.96(\mathrm{t}, J=5.9 \mathrm{~Hz}, 1 \mathrm{H}), 3.64(\mathrm{t}, J=7.0 \mathrm{~Hz}, 2 \mathrm{H}), 2.98(\mathrm{t}, J=7.0 \mathrm{~Hz}$ 2H), $2.43(\mathrm{~s}, 3 \mathrm{H}), 2.42-2.38(\mathrm{~m}, 4 \mathrm{H}), 1.75(\mathrm{bs}, 1 \mathrm{H}) ;{ }^{13} \mathrm{C} \mathrm{NMR}\left(151 \mathrm{MHz}, \mathrm{CDCl}_{3}\right) \delta 143.6,143.2,142.4$ 137.0, 131.4, 129.6, 129.5, 128.9, 128.8, 128.4, 128.2, 127.0, 126.6, 126.6, 61.0, 41.7, 35.2, 32.0, 21.6; LCMS (ES + APCI) $m / z:=422.3[\mathrm{M}+\mathrm{H}]^{+}$; HRMS (ESI-TOF) $m / z:[\mathrm{M}+\mathrm{H}]^{+}$calcd. for $\mathrm{C}_{25} \mathrm{H}_{28} \mathrm{NO}_{3} \mathrm{~S}$ 422.1790; found 422.1786 .

Oxidative heterocyclisation with probe compound (28). Reaction of $N$-(3-(diphenylmethylene)-5-hydroxypentyl)-4-methylbenzenesulfonamide 28 (170 $\mathrm{mg}, 0.4 \mathrm{mmol})$ and malonoyl peroxide 1 (77 $\mathrm{mg}, 0.8$ mmol) in HFIP (1.0 mL) according to General Procedure 5 to give a crude material. The crude was dissolved in $\mathrm{PhMe}(1.0 \mathrm{~mL})$ and $\mathrm{MeOH}(0.5 \mathrm{~mL})$ before the addition of $\mathrm{TMS}_{-} \mathrm{CHN}_{2}\left(2 \mathrm{M}\right.$ in $\mathrm{Et}_{2} \mathrm{O}, 1.0 \mathrm{~mL}$, $2.02 \mathrm{mmol}$ ) according to General Procedure 5. Purification by silica gel flash column chromatography (cyclohexane:EtOAc 3:1) afforded the cyclised furan compound $\mathbf{2 9}$ (104 mg, $0.2 \mathrm{mmol}$, 46\% over 2 steps) as a colourless gum and bicycle product (16 mg, $0.03 \mathrm{mmol}, 7 \%)$ as a colourless gum.

1-Methyl 1-(3-(2-((4-methylphenyl)sulfonamido)ethyl)-2,2-diphenyltetrahydrofuran-3-yl) cyclopropane1,1-dicarboxylate (29). IR (ATR)/cm ${ }^{-1}: 2954,1722,1438,1323 ;{ }^{1} \mathrm{H} \mathrm{NMR}\left(600 \mathrm{MHz}, \mathrm{CDCl}_{3}\right) \delta 7.63(\mathrm{t}, J$ $=7.5 \mathrm{~Hz}, 4 \mathrm{H}), 7.54(\mathrm{~d}, J=7.5 \mathrm{~Hz}, 2 \mathrm{H}), 7.31-7.16(\mathrm{~m}, 8 \mathrm{H}), 4.69(\mathrm{bs}, 1 \mathrm{H}), 4.12-4.02(\mathrm{~m}, 2 \mathrm{H}), 3.76(\mathrm{~s}$, $3 \mathrm{H}), 2.86-2.76(\mathrm{~m}, 1 \mathrm{H}), 2.77-2.64(\mathrm{~m}, 2 \mathrm{H}), 2.45(\mathrm{~s}, 3 \mathrm{H}), 2.36-2.29(\mathrm{~m}, 1 \mathrm{H}), 2.20(\mathrm{ddd}, J=15.0,8.0,6.5$ Hz, 1H), $2.06(\mathrm{dt}, J=15.0,7.5 \mathrm{~Hz}, 1 \mathrm{H}), 1.61-1.55(\mathrm{~m}, 1 \mathrm{H}), 1.49-1.45$ (m, 1H), 1.41-1.35 (m, 1H), $1.20-$ $1.14(\mathrm{~m}, 1 \mathrm{H}) ;{ }^{13} \mathrm{C} \mathrm{NMR}\left(151 \mathrm{MHz}, \mathrm{CDCl}_{3}\right) \delta(\mathrm{ppm}) 170.0,168.6,143.1,142.6,142.0,137.0,129.5,127.9$, $127.8,127.2,127.2,127.0,126.9,126.1,90.3,88.7,64.0,52.6,39.1,35.9,34.4,28.8,21.5,16.7,16.5$; LCMS (ES + APCI) $m / z:=586.3[\mathrm{M}+\mathrm{Na}]^{+} ; \mathrm{HRMS}(\mathrm{ESI}-\mathrm{TOF}) m / z:[\mathrm{M}+\mathrm{Na}]^{+}$calcd. for $\mathrm{C}_{31} \mathrm{H}_{33} \mathrm{NNaO}_{7} \mathrm{~S}$ 586.1875; found 586.1873.

Methyl 1-(5-(2-((4-methylphenyl)sulphonamide)ethyl)-6,6-diphenyl-2,7,8-trioxabicyclo[3.2.1]octan-1yl)cyclopropane-1-carboxylate (31). IR (ATR)/cm ${ }^{-1}: 2954,1723,1328,1161,1040 ;{ }^{1} \mathrm{H}$ NMR (600 MHz, $\left.\mathrm{CDCl}_{3}\right) \delta 7.75(\mathrm{~d}, J=7.9 \mathrm{~Hz}, 2 \mathrm{H}), 7.41-7.37(\mathrm{~m}, 2 \mathrm{H}), 7.32-7.21(\mathrm{~m}, 8 \mathrm{H}), 5.14(\mathrm{dd}, J=7.3,3.2 \mathrm{~Hz}, 1 \mathrm{H})$, $3.85(\mathrm{~s}, 3 \mathrm{H}), 3.75-3.67(\mathrm{~m}, 1 \mathrm{H}), 3.58(\mathrm{dd}, J=11.3,6.8 \mathrm{~Hz}, 1 \mathrm{H}), 3.14-3.07(\mathrm{~m}, 1 \mathrm{H}), 2.99(\mathrm{dt}, J=8.6,4.2$ 
$\mathrm{Hz}, 1 \mathrm{H}), 2.45(\mathrm{~s}, 3 \mathrm{H}), 2.00(\mathrm{td}, J=12.9,7.0 \mathrm{~Hz}, 1 \mathrm{H}), 1.92-1.87(\mathrm{~m}, 1 \mathrm{H}), 1.82-1.74(\mathrm{~m}, 1 \mathrm{H}), 1.54(\mathrm{dd}, J$

$=13.6,3.8 \mathrm{~Hz}, 1 \mathrm{H}), 1.50-1.45(\mathrm{~m}, 1 \mathrm{H}), 1.41-1.32(\mathrm{~m}, 3 \mathrm{H}) ;{ }^{13} \mathrm{C} \mathrm{NMR}\left(151 \mathrm{MHz}, \mathrm{CDCl}_{3}\right) \delta 171.7,143.3$, $141.1,139.8,136.8,129.7,128.5,127.8,127.7,127.7,127.1,118.1,90.7,86.1,59.2,52.5,39.3,35.1$, 29.3, 29.1, 21.5, 14.6, 13.4; LCMS (ES + APCI) $m / z:=586.3[\mathrm{M}+\mathrm{Na}]^{+}$; HRMS (ESI-TOF) $m / z:[\mathrm{M}+\mathrm{Na}]^{+}$ calcd. for $\mathrm{C}_{31} \mathrm{H}_{33} \mathrm{NNaO}_{7} \mathrm{~S} 586.1875$; found 586.1972.

General Procedure 4: Mitsunobu reaction. A dry three-neck flask was charged with cinnamyl alcohol (1.0 equiv), $\mathrm{PPh}_{3}$ (1.1 equiv) and $N$-hydroxyphthalimide (1.1 equiv) in anhydrous THF (0.25 M). The solution was cooled to $0^{\circ} \mathrm{C}$ and diethyl azodicarboxylate (2.2 $\mathrm{M}$ in $\mathrm{PhMe}, 1.1$ equiv) was added dropwise. The mixture was warmed to $\mathrm{rt}$ and stirred for $2.5 \mathrm{~h}$. The solvent was removed by rotary evaporation before purification by silica gel flash column chromatography with hexane:EtOAc mixtures afforded the target compound.

2-(Cinnamyloxy)isoindoline-1,3-dione. ${ }^{36}$ Cinnamyl alcohol (2.2 g, $\left.16.0 \mathrm{mmol}\right), \mathrm{PPh}_{3}(4.6 \mathrm{~g}, 17.6 \mathrm{mmol})$ and $N$-hydroxyphthalimide $(2.9 \mathrm{~g}, 17.6 \mathrm{mmol})$ were dissolved in anhydrous THF $(64 \mathrm{~mL})$ before the dropwise addition of diethyl azodicarboxylate $(2.2 \mathrm{M}$ in PhMe, $8.0 \mathrm{~mL}, 17.6 \mathrm{mmol})$ according to the General Procedure 4. Purification by silica gel flash column chromatography (hexane:EtOAc 8:2) afforded the title compound $(4.3 \mathrm{~g}, 15.4 \mathrm{mmol}, 96 \%)$ as a white solid. m.p. $148-150{ }^{\circ} \mathrm{C}, \mathrm{Lit}^{37}\left[116-118^{\circ} \mathrm{C}\right]$; IR (ATR)/ $\mathrm{cm}^{-1}: 3058,3028,2948,1790 ;{ }^{1} \mathrm{H}$ NMR (500 MHz, $\left.\mathrm{CDCl}_{3}\right) \delta 7.82-7.80(\mathrm{~m}, 2 \mathrm{H}), 7.74-7.71$ (m, 2H), 7.39-7.37 (m, 2H), 7.32-7.29 (m, 2H), 7.27-7.24 (m, 1H), $6.67(\mathrm{~d}, J=15.9 \mathrm{~Hz}, 1 \mathrm{H}), 6.47(\mathrm{dt}$, $J=15.9,7.1 \mathrm{~Hz}, 1 \mathrm{H}), 4.87(\mathrm{~d}, J=7.1 \mathrm{~Hz}, 2 \mathrm{H}) ;{ }^{13} \mathrm{C} \mathrm{NMR}\left(126 \mathrm{MHz}, \mathrm{CDCl}_{3}\right) \delta 164.0,137.7,136.0,134.6$, 129.0, 128.8, 128.6, 127.1, 123.7, 122.2, 78.8; LRMS (ES + APCI) $m / z: 297.0\left[\mathrm{M}+\mathrm{NH}_{4}\right]^{+}$.

(E)-2-((3-(4-Chlorophenyl)allyl)oxy)isoindoline-1,3-dione. (E)-3-(4-Chlorophenyl)prop-2-en-1-ol (300 $\mathrm{mg}, 1.8 \mathrm{mmol}), \mathrm{PPh}_{3}(513 \mathrm{mg}, 2.0 \mathrm{mmol})$ and $N$-hydroxyphthalimide $(320 \mathrm{mg}, 2.0 \mathrm{mmol})$ were dissolved in anhydrous THF (7.0 mL) before the dropwise addition of diethyl azodicarboxylate (2.2 $\mathrm{M}$ in PhMe, $0.9 \mathrm{~mL}, 1.96 \mathrm{mmol}$ ) according to General Procedure 4. Purification by silica gel flash column chromatography (hexane:EtOAc 8:2) afforded the title compound (200 mg, $0.6 \mathrm{mmol}, 35 \%$ ) as a white solid. m.p. 
$138-140{ }^{\circ} \mathrm{C}$; IR (ATR)/cm ${ }^{-1}: 3050,2948,1788,1742 ;{ }^{1} \mathrm{H}$ NMR $\left(400 \mathrm{MHz}, \mathrm{CDCl}_{3}\right) \delta 7.83-7.81(\mathrm{~m}, 2 \mathrm{H})$, 7.74-7.72 (m, 2H), 7.32-7.27 (m, 4H), $6.63(\mathrm{~d}, J=15.9 \mathrm{~Hz}, 1 \mathrm{H}), 6.44(\mathrm{dt}, J=15.9,7.0 \mathrm{~Hz}, 1 \mathrm{H}), 4.85(\mathrm{~d}$, $J=7.0,2 \mathrm{H}) ;{ }^{13} \mathrm{C} \mathrm{NMR}\left(101 \mathrm{MHz}, \mathrm{CDCl}_{3}\right) \delta 163.8,136.0,134.5,134.3,134.2,128.9,128.8,128.1,123.5$, 122.8, 78.4; LRMS (ES + APCI) $m / z: 314.0[\mathrm{M}+\mathrm{H}]^{+}$; HRMS (ESI-TOF) $m / z:[\mathrm{M}+\mathrm{H}]^{+}$calcd. for $\mathrm{C}_{17} \mathrm{H}_{13}{ }^{35} \mathrm{ClNO}_{3} 314.0584$; found 314.0583 .

$N$-(Cinnamyloxy)-4-methylbenzenesulfonamide (33). ${ }^{38}$ To a solution of 2-(cinnamyloxy)isoindoline-1,3dione $(4.5 \mathrm{~g}, 16.1 \mathrm{mmol})$ in EtOH $(62 \mathrm{~mL})$ was added hydrazine monohydrate $(1.6 \mathrm{~mL}, 33.8 \mathrm{mmol})$ according to General Procedure 2 to give $O$-cinnamylhydroxylamine.

To a solution of crude $O$-cinnamylhydroxylamine $(16.1 \mathrm{mmol})$ in $\mathrm{CH}_{2} \mathrm{Cl}_{2}(65 \mathrm{~mL})$ was added $\mathrm{Et}_{3} \mathrm{~N}$ (27 $\mathrm{mL}, 19.3 \mathrm{mmol})$ followed by $p$-TsCl $(3.4 \mathrm{~g}, 17.7 \mathrm{mmol})$ and DMAP (0.6 g, $4.8 \mathrm{mmol})$ according to General Procedure 2. Purification by silica gel flash column chromatography (hexane:EtOAc 8:2) afforded the title compound 33 (2.9 g, $9.6 \mathrm{mmol}, 60 \%)$ as a white solid. m.p. $109-110{ }^{\circ} \mathrm{C}$, $\mathrm{Lit}^{37}\left[103{ }^{\circ} \mathrm{C}\right]$; IR $(\mathrm{ATR}) / \mathrm{cm}^{-1}: 3220,3058,3026,2924,2870 ;{ }^{1} \mathrm{H} \mathrm{NMR}\left(500 \mathrm{MHz}, \mathrm{CDCl}_{3}\right) \delta 7.83(\mathrm{~d}, J=8.3 \mathrm{~Hz}, 2 \mathrm{H}), 7.38-$ $7.25(\mathrm{~m}, 7 \mathrm{H}), 6.91(\mathrm{bs}, 1 \mathrm{H}), 6.63(\mathrm{~d}, J=15.9 \mathrm{~Hz}, 1 \mathrm{H}), 6.22(\mathrm{dt}, J=15.9,6.8 \mathrm{~Hz}, 1 \mathrm{H}), 4.61(\mathrm{~d}, J=6.8$, 2H), $2.44(\mathrm{~s}, 3 \mathrm{H}) ;{ }^{13} \mathrm{C} \mathrm{NMR}\left(126 \mathrm{MHz}, \mathrm{CDCl}_{3}\right) \delta 145.1,136.3,136.0,133.8,129.9,128.77,128.75,128.4$, 126.9, 122.9, 78.1, 21.8; LRMS (ES + APCI) $m / z: 321.0\left[\mathrm{M}+\mathrm{NH}_{4}\right]^{+}$.

(E)-N-((3-(4-Chlorophenyl)allyl)oxy)-4-methylbenzenesulfonamide (35). To a solution of (E)-2-((3-(4chlorophenyl)allyl)oxy)isoindoline-1,3-dione (202 mg, $0.6 \mathrm{mmol})$ in EtOH (2.3 mL) was added hydrazine monohydrate $(61 \mu \mathrm{L}, 1.3 \mathrm{mmol})$ according to General Procedure 2 to give $(E)-O$-(3-(4-chlorophenyl)allyl)hydroxylamine.

To a solution of crude $(E)-O$-(3-(4-chlorophenyl)allyl)hydroxylamine $(0.6 \mathrm{mmol})$ in $\mathrm{CH}_{2} \mathrm{Cl}_{2}(2.2 \mathrm{~mL})$ was added $\mathrm{Et}_{3} \mathrm{~N}(120 \mu \mathrm{L}, 0.8 \mathrm{mmol})$ followed by $p$-TsCl $(107 \mathrm{mg}, 0.6 \mathrm{mmol})$ and DMAP (22 mg, $\left.0.2 \mathrm{mmol}\right)$ according to General Procedure 2. Purification by silica gel flash column chromatography (hexane:EtOAc 8:2) afforded the title compound 35 (102 mg, $0.3 \mathrm{mmol}, 54 \%)$ as a white solid. m.p. $116-118{ }^{\circ} \mathrm{C}$; IR (ATR) $/ \mathrm{cm}^{-1}: 3216,3064,2922,2852 ;{ }^{1} \mathrm{H}$ NMR $\left(500 \mathrm{MHz}, \mathrm{CDCl}_{3}\right) \delta 7.80(\mathrm{~d}, J=8.3 \mathrm{~Hz}, 2 \mathrm{H}), 7.31(\mathrm{~d}, J$ 
$=8.0 \mathrm{~Hz}, 2 \mathrm{H}), 7.27-7.25(\mathrm{~s}, 4 \mathrm{H}), 7.04(\mathrm{bs}, 1 \mathrm{H}), 6.54(\mathrm{~d}, J=15.9 \mathrm{~Hz}, 1 \mathrm{H}), 6.17(\mathrm{dt}, J=15.9,6.7 \mathrm{~Hz}, 1 \mathrm{H})$,

$4.57(\mathrm{bd}, J=6.7 \mathrm{~Hz}, 2 \mathrm{H}), 2.41(\mathrm{~s}, 3 \mathrm{H}) ;{ }^{13} \mathrm{C} \mathrm{NMR}\left(126 \mathrm{MHz}, \mathrm{CDCl}_{3}\right) \delta 145.0,134.7,134.4,133.9,133.7$, 129.8, 128.8, 128.6, 127.9, 123.6, 77.6, 21.7; LRMS (ES + APCI) $m / z: 355.0\left[\mathrm{M}+\mathrm{NH}_{4}\right]^{+}$; HRMS (ESI-TOF) $m / z:[\mathrm{M}+\mathrm{H}]^{+}$calcd. for $\mathrm{C}_{16} \mathrm{H}_{17}{ }^{35} \mathrm{ClNO}_{3} \mathrm{~S} 338.0618$; found 338.0616.

General Procedure 5: Oxidative cyclisation for the synthesis of isoxazolidines. Malonoyl peroxide 1 (1.5 equiv) was added to a solution of alkene 33 or 34 (1.0 equiv) in HFIP (0.5 M). The mixture was stirred at $40{ }^{\circ} \mathrm{C}$ for $18 \mathrm{~h}$ before removal of the solvent by rotary evaporation. The residue was dissolved in $\mathrm{PhMe}(0.2 \mathrm{M})$ and $\mathrm{MeOH}(0.5 \mathrm{M})$ and a solution of $\mathrm{TMS}^{-} \mathrm{CHN}_{2}$ in $\mathrm{Et}_{2} \mathrm{O}$ (2.0 equiv) was added dropwise. The resulting mixture was stirred at $\mathrm{rt}$ for $2 \mathrm{~h}$ before the solvents were evaporated under reduced pressure. Purification of the crude material by silica gel flash column chromatography with petroleum ether $\left(40-60{ }^{\circ} \mathrm{C}\right): \mathrm{Et}_{2} \mathrm{O}$ mixtures afforded the target compounds.

(土)-1-(((-3-Phenyl-2-tosylisoxazolidin-4-yl)oxy)carbonyl)cyclopropane-1-carboxylic acid (37). To a solution of $N$-(cinnamyloxy)-4-methylbenzenesulfonamide $33(151 \mathrm{mg}, 0.50 \mathrm{mmol})$ in HFIP $(1.0 \mathrm{~mL})$ was added malonoyl peroxide 1 (96 $\mathrm{mg}, 0.75 \mathrm{mmol}$ ) according to General Procedure 5 (without the TMS$\mathrm{CHN}_{2}$ methyl ester formation) to give crude isoxazolidine (1:10 cis:trans). Purification by silica gel flash column chromatography (EtOAc then EtOAc:AcOH 0.5\%) afforded the title compound 37 (178 mg, 0.41 mmol, 83\%) as a white solid for characterisation and X-ray analysis purposes. m.p. $137-139{ }^{\circ} \mathrm{C}$; IR $(\mathrm{ATR}) / \mathrm{cm}^{-1}: 3550,2930,2852,1716,1660 ;{ }^{1} \mathrm{H} \mathrm{NMR}\left(500 \mathrm{MHz}, \mathrm{CDCl}_{3}\right) \delta 12.19(\mathrm{bs}, 1 \mathrm{H}), 7.88(\mathrm{~d}, J=$ $8.3 \mathrm{~Hz}, 2 \mathrm{H}), 7.44-7.34(\mathrm{~m}, 7 \mathrm{H}), 5.61(\mathrm{ddd}, J=6.0,3.1,0.7 \mathrm{~Hz}, 1 \mathrm{H}), 5.54(\mathrm{bs}, 1 \mathrm{H}), 4.44(\mathrm{dd}, J=9.6,3.1$ Hz, 1H), 4.39 (dd, $J=9.6,6.0 \mathrm{~Hz}, 1 \mathrm{H}), 2.47$ (s, 3H), 2.18-2.14 (m, 1H), 2.06-2.03 (m, 1H), 1.99-1.93 $(\mathrm{m}, 2 \mathrm{H}) ;{ }^{13} \mathrm{C} \mathrm{NMR}\left(126 \mathrm{MHz}, \mathrm{CDCl}_{3}\right) \delta 176.3,170.0,145.5,136.3,133.7,129.9,129.2,129.1,128.7$, 126.5, 84.9, 74.6, 66.9, 25.6, 23.5, 23.2, 21.9; LRMS (ES + APCI) m/z: $432.0[\mathrm{M}+\mathrm{H}]^{+}$; HRMS (ESI-TOF) $m / z:[\mathrm{M}-\mathrm{H}]^{-}$calcd. for $\mathrm{C}_{21} \mathrm{H}_{20} \mathrm{NO}_{7} \mathrm{~S} 430.0960$; found 430.0954 .

(士)-1-Methyl 1-((3R,4S)-3-phenyl-2-tosylisoxazolidin-4-yl) cyclopropane-1,1-dicarboxylate (34). To a solution of $N$-(cinnamyloxy)-4-methylbenzenesulfonamide 33 (185 mg, $0.43 \mathrm{mmol})$ in HFIP (0.9 mL) was 
added malonoyl peroxide $\mathbf{1}(82 \mathrm{mg}, 0.65 \mathrm{mmol}$ ) according to General Procedure 5 to give crude isoxazolidine (1:10 cis:trans) which was dissolved in $\mathrm{PhMe}(2.2 \mathrm{~mL})$ and $\mathrm{MeOH}(0.9 \mathrm{~mL})$ before the addition of $\mathrm{TMS}_{-\mathrm{CHN}_{2}}\left(2 \mathrm{M}\right.$ in $\left.\mathrm{Et}_{2} \mathrm{O}, 0.43 \mathrm{~mL}, 0.86 \mathrm{mmol}\right)$ according to General Procedure 5. Purification by silica gel flash column chromatography (petroleum ether $\left(40-60^{\circ} \mathrm{C}\right)$ :EtOAc 6:4) afforded the title compound 34 (154 mg, $0.35 \mathrm{mmol}, 80 \%$ over two steps) as a colourless oil. IR (ATR)/cm ${ }^{-1}: 3028,3062,2954,1727$; ${ }^{1} \mathrm{H}$ NMR $\left(500 \mathrm{MHz}, \mathrm{CDCl}_{3}\right) \delta 7.89(\mathrm{~d}, J=8.3 \mathrm{~Hz}, 2 \mathrm{H}), 7.47(\mathrm{~d}, J=7.3 \mathrm{~Hz}, 2 \mathrm{H}), 7.38-7.35(\mathrm{~m}, 4 \mathrm{H}), 7.31$ (t, $J=7.3 \mathrm{~Hz}, 1 \mathrm{H}), 5.52(\mathrm{ddd}, J=6.1,4.1,1.7 \mathrm{~Hz}, 1 \mathrm{H}), 5.56(\mathrm{bs}, 1 \mathrm{H}), 4.38(\mathrm{dd}, J=9.3,6.1 \mathrm{~Hz}, 1 \mathrm{H})$, $4.34(\mathrm{dd}, J=9.3,4.1 \mathrm{~Hz}, 1 \mathrm{H}), 3.80(\mathrm{~s}, 3 \mathrm{H}), 2.46(\mathrm{~s}, 3 \mathrm{H}), 1.67-1.51(\mathrm{~m}, 4 \mathrm{H}) ;{ }^{13} \mathrm{C} \mathrm{NMR}\left(126 \mathrm{MHz}, \mathrm{CDCl}_{3}\right)$ $\delta 169.9,169.4,145.2,137.3,133.9,129.8,129.2,129.0,128.3,126.6,83.8,74.4,67.0,53.0,27.9,21.9$, 17.6, 17.5; LRMS (ES + APCI) $m / z: 463.0\left[\mathrm{M}+\mathrm{NH}_{4}\right]^{+}$; HRMS (ESI-TOF) $m / z:[\mathrm{M}+\mathrm{H}]^{+}$calcd. for $\mathrm{C}_{22} \mathrm{H}_{24} \mathrm{NO}_{7} \mathrm{~S}$ 446.1273; found 446.1266.

(土)-1-((3R,4S)-3-(4-Chlorophenyl)-2-tosylisoxazolidin-4-yl) 1-methylcyclopropane-1,1-dicarboxylate

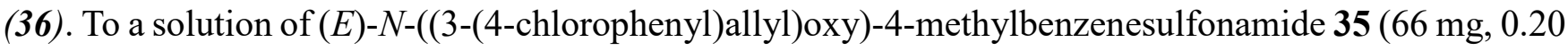
mmol) in HFIP (0.4 mL) was added malonoyl peroxide 1 (38 mg, $0.29 \mathrm{mmol})$ according to General Procedure 5 to give crude isoxazolidine (1:7 cis:trans) which was dissolved in PhMe (1.0 mL) and $\mathrm{MeOH}$ $(0.4 \mathrm{~mL})$ before the addition of $\mathrm{TMS}^{-\mathrm{CHN}_{2}}\left(2 \mathrm{M}\right.$ in $\left.\mathrm{Et}_{2} \mathrm{O}, 0.2 \mathrm{~mL}, 0.40 \mathrm{mmol}\right)$ according to General Procedure 5. Purification by silica gel flash column chromatography (petroleum ether $\left(40-60{ }^{\circ} \mathrm{C}\right)$ :EtOAc 6:4) afforded the title compound $\mathbf{3 6}$ (80 $\mathrm{mg}, 0.17 \mathrm{mmol}, 85 \%$ over 2 steps) as a white solid. m.p. 110-112 ${ }^{\circ} \mathrm{C}$; IR (ATR)/cm ${ }^{-1}: 3040,2930,2904,1719,1697 ;{ }^{1} \mathrm{H} \mathrm{NMR}\left(500 \mathrm{MHz}, \mathrm{CDCl}_{3}\right) \delta 7.87(\mathrm{~d}, J=8.3 \mathrm{~Hz}$, 2H), $7.42(\mathrm{~d}, J=8.5 \mathrm{~Hz}, 2 \mathrm{H}), 7.36(\mathrm{~d}, J=8.3 \mathrm{~Hz}, 2 \mathrm{H}), 7.33(\mathrm{~d}, J=8.5 \mathrm{~Hz}, 2 \mathrm{H}), 5.47-5.45(\mathrm{~m}, 1 \mathrm{H}), 5.41$ (bs, 1H), 4.35-4.34 (m, 2H), $3.79(\mathrm{~s}, 3 \mathrm{H}), 2.46(\mathrm{~s}, 3 \mathrm{H}), 1.66-1.52(\mathrm{~m}, 4 \mathrm{H}) ;{ }^{13} \mathrm{C} \mathrm{NMR}\left(126 \mathrm{MHz}, \mathrm{CDCl}_{3}\right)$ $\delta 169.8,169.5,145.3,135.8,134.3,133.6,129.9,129.2,129.1,128.0,83.7,74.2,66.5,53.0,27.9,21.9$, 17.6, 17.5; LRMS (ES + APCI) $m / z: 497.0\left[\mathrm{M}+\mathrm{NH}_{4}\right]^{+}$; HRMS (ESI-TOF) $m / z:[\mathrm{M}+\mathrm{H}]^{+}$calcd. for $\mathrm{C}_{22} \mathrm{H}_{23}{ }^{35} \mathrm{ClNO}_{7} \mathrm{~S} 480.0889$; found 480.0879 . 
(士)-2-Phenyl-1-tosylpyrrolidin-3-one (38). ${ }^{39}$ (2R,3S)-2-Phenyl-1-tosylpyrrolidin-3-ol 9a (100 mg, 0.32 mmol) was dissolved in degassed MeCN (1.6 mL). IBX (265 mg, $0.95 \mathrm{mmol})$ was added and the mixture was stirred at $80^{\circ} \mathrm{C}$ for $18 \mathrm{~h}$. The mixture was filtered through Celite ${ }^{\circledR}$ and the solvent removed under reduced pressure. Purification by silica gel flash column chromatography (hexane:EtOAc 4:6) afforded the title compound $\mathbf{3 8}(84 \mathrm{mg}, 0.27 \mathrm{mmol}, 85 \%)$ as a colourless oil. m.p. $124-126^{\circ} \mathrm{C}, \mathrm{Lit}^{40}\left[140-141^{\circ} \mathrm{C}\right]$; IR (ATR) $/ \mathrm{cm}^{-1}: 2950,2924,2855,1753 ;{ }^{1} \mathrm{H}$ NMR (500 MHz, $\left.\mathrm{CDCl}_{3}\right) \delta 7.57(\mathrm{~d}, J=8.2 \mathrm{~Hz}, 2 \mathrm{H}), 7.32-$ $7.27(\mathrm{~m}, 7 \mathrm{H}), 4.59(\mathrm{~s}, 1 \mathrm{H}), 3.94(\mathrm{ddd}, J=10.6,9.0,5.6 \mathrm{~Hz}, 1 \mathrm{H}), 3.72(\mathrm{dt}, J=10.7,8.1 \mathrm{~Hz}, 1 \mathrm{H}), 2.63-$ $2.55(\mathrm{~m}, 1 \mathrm{H}), 2.49-2.42(\mathrm{~m}, 1 \mathrm{H}), 2.42(\mathrm{~s}, 3 \mathrm{H}) ;{ }^{13} \mathrm{C} \mathrm{NMR}\left(126 \mathrm{MHz}, \mathrm{CDCl}_{3}\right) \delta 208.3,144.4,135.6,133.9$, 130.0, 128.8, 128.4, 127.8, 127.0, 67.3, 44.2, 35.9, 21.7; LRMS (ES + APCI) m/z: $316.1[\mathrm{M}+\mathrm{H}]^{+}$.

( \pm )-2-Phenyl-1-tosylpyrrolidin-3-ol (22). ${ }^{39}$ To a cooled $\left(0{ }^{\circ} \mathrm{C}\right)$ solution of $( \pm)$-2-phenyl-1-tosylpyrrolidin3-one 38 (64 mg, $0.20 \mathrm{mmol})$ in THF (1 mL) was added a solution of DIBAL-H (1 M in THF, $0.3 \mathrm{~mL}$, $0.30 \mathrm{mmol})$. The mixture was allowed to warm to room temperature and stirred for $3 \mathrm{~h}$, before quenching with a $2 \mathrm{M}$ solution of $\mathrm{HCl}(5 \mathrm{~mL})$ and diluted with EtOAc $(5 \mathrm{~mL})$. The organic layer was separated and further extracted with EtOAc $(3 \times 10 \mathrm{~mL})$. The combined organics were washed with brine $(50 \mathrm{~mL})$, dried over $\mathrm{MgSO}_{4}$, filtered and the solvent was removed under reduced pressure affording the title compound $22(50 \mathrm{mg}, 0.16 \mathrm{mmol}, 78 \%)$ as a colourless oil as a diastereomeric mixture (6:1 cis:trans). ${ }^{1} \mathrm{H}$ NMR (400 $\left.\mathrm{MHz}_{\mathrm{CDCl}}\right) \delta$ 7.69-7.64 (m, 2H), 7.39-7.28 (m, 7H), $4.74(\mathrm{~d}, J=5.6 \mathrm{~Hz}, 1 \mathrm{H}), 4.25-4.15(\mathrm{~m}, 1 \mathrm{H}), 3.80-$ $3.70(\mathrm{~m}, 1 \mathrm{H}), 3.64(\mathrm{ddd}, J=10.7,7.7,4.9 \mathrm{~Hz}, 1 \mathrm{H}), 2.43(\mathrm{~s}, 3 \mathrm{H}), 1.89(\mathrm{ddt}, J=16.3,6.5,4.9 \mathrm{~Hz}, 1 \mathrm{H})$, 1.80-1.69 (m, 1H), $1.09(\mathrm{~d}, \mathrm{~J}=4.9 \mathrm{~Hz}, 1 \mathrm{H})$.

\section{ASSOCIATED CONTENT}

\section{Supporting Information}

NMR spectra for all compounds reported, X-ray data for 10 and $\mathbf{3 6}$ and DSC data for $\mathbf{1}$ (PDF).

\section{AUTHOR INFORMATION}

\section{Corresponding Author}


*E-Mail: Nicholas.Tomkinson@strath.ac.uk

\section{ORCID}

Nicholas C. O. Tomkinson: 0000-0002-5509-0133

\section{ACKNOWLEDGMENT}

The authors thank Sean Lynn for NMR characterization of 31, the EPSRC and GlaxoSmithKline for financial support and the EPSRC Mass Spectrometry Service, Swansea, for high-resolution spectra.

\section{REFERENCES}

(1) Kolb, H. C.; VanNieuwenhze, M. S.; Sharpless, K. B. Chem. Rev. 1994, 94, 2483-2547.

(2) For reviews on transition metal catalyzed dihydroxylation see: (a) Bataille, C. J. R.; Donohoe, T. J. Chem. Soc. Rev. 2011, 40, 114-128. (b) Asghar, S. F.; Lewis, S. E. Annu. Rep. Prog. Chem., Sect. B: Org. Chem. 2011, 107, 34-67. (c) Christie, S. D. R.; Warrington, A. D. Synthesis 2008, 1325-1341.

(3) For a review on metal free dihydroxylation see: Rawling M. J.; Tomkinson, N. C. O. Org. Biomol. Chem. 2013, 11, 1434-1440.

(4) For reviews on the aminohydroxylation reaction see: (a) Bodkin, J. A.; McLeod, M. D. J. Chem. Soc., Perkin Trans. 1 2002, 2733-2746. (b) Muniz, K. Chem. Soc. Rev. 2004, 33, 166-174. (c) Knappke, C. E. I.; von Wangelin, A. J. ChemCatChem 2010, 2, 1381-1383. (d) Donohoe, T. J.; Callens, C. K. A.; Flores, A.; Lacy, A. R.; Rathi, A. H. Chem. Eur. J. 2011, 17, 58-76.

(5) (a) O’Brien, P. Angew. Chem., Int. Ed. Engl. 1999, 38, 326-329. (b) Li, G.; Chang, H.-T.; Sharpless, K. B. Angew. Chem., Int. Ed. Engl. 1996, 35, 451-454.

(6) For selected examples see: (a) Streuff, J.; Osterath, B.; Nieger, M.; Muñiz, K. Tetrahedron: Asymmetry 2005, 16, 3492-3496. (b) Harding, M.; Bodkin, J. A.; Hutton, C. A.; McLeod, M. D. Synlett 2005, 28292831. (c) Donohoe, T. J.; Johnson, P. D.; Helliwell, M.; Keenan, M. Chem. Commun. 2001, 2078-2079. (d) Donohoe, T. J.; Johnson, P. D.; Cowley, A.; Keenan, M. J. Am. Chem. Soc. 2002, 124, 12934-12935. 
(e) Donohoe, T. J.; Butterworth, S. Angew. Chem. Int. Ed. Engl. 2003, 42, 948-951. (f) Donohoe, T. J.;

Churchill, G. H.; Wheelhouse, K. M. P.; Glossop, P. A. Angew. Chem. Int. Ed. 2006, 45, 8025-8028.

(7) For example see: (a) Levites-Agababa, E.; Menhaji, E.; Perlson, L. N.; Rojas, C. M. Org. Lett. 2002, 4, 863-865. (b) Espino, C. G.; Wehn, P. M.; Chow, J.; Du Bois, J. J. Am. Chem. Soc. 2001, 123, 69356936. (c) Padwa, A.; Stengel, T. Org. Lett. 2002, 4, 2137-2139. (d) Beaumont, S.; Pons, V.; Retailleau, P.; Dodd, R. H.; Dauban, P. Angew. Chem. Int. Ed. 2010, 49, 1634-1637. (e) Lorpitthaya, R.; Sophy, K. B.; Kuo, J.-L.; Liu, X.-W. Org. Biomol. Chem. 2009, 7, 1284-1287.

(8) For example see: (a) Bäckvall, J.-E. Tetrahedron Lett. 1975, 16, 2225-2228. (b) Alexanian, E. J.; Lee, C.; Sorensen, E. J. J. Am. Chem. Soc. 2005, 127, 7690-7691. (c) Liu, G.; Stahl, S. S. J. Am. Chem. Soc. 2006, 128, 7179-7181.

(9) For example see: (a) Noack, M.; Gottlich, R. Chem. Commun. 2002, 536-537. (b) Fuller, P. H.; Kim, J.-W.; Chemler, S. R. J. Am. Chem. Soc. 2008, 130, 17638-17639. (c) Michaelis, D. J.; Shaffer, C. J.; Yoon, T. P. J. Am. Chem. Soc. 2007, 129, 1866-1867.

(10) For example see: (a) Williamson, K. S.; Yoon, T. P. J. Am. Chem. Soc. 2010, 132, 4570-4571. (b) Williamson, K. S.; Yoon, T. P. J. Am. Chem. Soc. 2012, 134, 12370-12373. (c) Liu, G.-S.; Zhang, Y.-Q.; Yuan, Y.-A.; Xu, H. J. Am. Chem. Soc. 2013, 135, 3343-3346. (d) Lu, D.-F.; Zhu, C.-L.; Jia, Z.-X.; Xu, H. J. Am. Chem. Soc. 2014, 136, 13186-13189.

(11) For example see: Dangerfield, E. M.; Timmer, M. S. M.; Stocker, B. L. Org. Lett. 2009, 11, 535-538. (12) For example see: (a) Serna, S.; Tellitu, I.; Domínguez, E.; Moreno, I.; SanMartin, R. Tetrahedron 2004, 60, 6533-6539. (b) Wardrop, D. J.; Bowen, E. G.; Forslund, R. E.; Sussman, A. D.; Weerasekera, S. L. J. Am. Chem. Soc. 2010, 132, 1188-1189. (c) Cochran, B. M.; Michael, F. E. Org. Lett. 2008, 10, 5039-5042. (d) Lovick, H. M.; Michael, F. E. J. Am. Chem. Soc. 2010, 132, 1249-1251.

(13) (a) Griffith, J. C.; Jones, K. M.; Picon, S.; Rawling, M. J.; Kariuki, B. M.; Campbell, M.; Tomkinson, N. C. O. J. Am. Chem. Soc. 2010, 132, 14409-14411. (b) Jones, K. M.; Tomkinson, N. C. O. J. Org. Chem. 2012, 77, 921-928. (c) Picon, S.; Rawling, M.; Campbell, M.; Tomkinson, N. C. O. Org. Lett. 2012, 14, $6250-6253$. 
(14) Alamillo-Ferrer, C.; Davidson, S. C.; Rawling, M. J.; Theodoulou, N. H.; Campbell, M.; Humphreys, P. G.; Kennedy, A. R.; Tomkinson, N. C. O. Org. Lett. 2015, 17, 5132-5135.

(15) Alamillo-Ferrer, C.; Karabourniotis-Sotti, M.; Kennedy, A. R.; Campbell, M.; Tomkinson, N. C. O. Org. Lett. 2016, 18, 3102-3105.

(16) Rawling, M. J.; Rowley, J. H.; Campbell, M.; Kennedy, A. R.; Parkinson, J. A.; Tomkinson, N. C. O. Chem. Sci. 2014, 5, 1777-1785.

(17) Kuznetsov, N. Y.; Tikhov, R. M.; Godovikov, I. A.; Khrustalev, V. N.; Bubnov, Y. N. Org. Biomol. Chem. 2016, 14, 4283-4298.

(18) Chen, X.-M.; Ning, X.-S.; Kang, Y.-B. Org. Lett. 2016, 18, 5368-5371.

(19) de Meijere, A.; Meyer, F. E. Angew. Chem. Int. Ed. Engl. 1994, 33, 2379-2411.

(20) Weidner-Wells, M. A.; Oda, K.; Mazzocchi, P. H. Tetrahedron 1997, 53, 3475-3486.

(21) Schlummer, B.; Hartwig, J. F. Org. Lett. 2002, 4, 1471-1474.

(22) McEven, W. E.; Cooney, J. V. J. Org. Chem. 1983, 48, 983-987.

(23) Agouridas, C.; Denis, A.; Auger, J.-M.; Benedetti, Y.; Bonnefoy, A.; Bretin, F.; Chantot, J.-F.; Dussarat, A.; Fromentin, C.; D'Ambrières, S. G.; Lachaud, S.; Laurin, P.; Le Martret, O.; Loyau, V.; Tessot, N. J. Med. Chem. 1998, 41, 4080-4100.

(24) Harusawa, S.; Sawada, K.; Magata, T.; Yoneyama, H.; Araki, L.; Usami, Y.; Hatano, K.; Yamamoto, K.; Yamamoto, D.; Yamatodani, A. Bioorg. Med. Chem. Lett. 2013, 23, 6415-6420.

(25) Zeng, X.; Miao, C.; Wang, S.; Xia, C.; Sun, W. Chem. Commun. 2013, 49, 2418-2420.

(26) Saikia, I.; Borah, A. J.; Phukan, P. Chem. Rev. 2016, 116, 6837-7042.

(27) Van der Bent, A.; Blommaert, A. G. S.; Melman, C. T. M.; Ijzerman, A. P.; Van Wijngaarden, I.; Soudijn, W. J. Med. Chem. 1992, 35, 1042-1049.

(28) Hamaguchi, F. Chem. Abstr. 1963, 58, 4492.

(29) Leger, P. R.; Murphy, R. A.; Pushkarskaya, E.; Sarpong, R. Chem. Eur. J. 2015, 21, 4377-4383.

(30) Tamaru, Y.; Hojo, M.; Kawamura, S.; Yoshida, Z. J. Org. Chem., 1986, 51, 4089-4090. 
(31) Ciesielski, J.; Dequirez, G.; Retailleau, P.; Gandon, V.; Dauban, P. Chem. Eur. J. 2016, 22, 93389347.

(32) Sugasawa, S.; Ushioda, S. Tetrahedron 1959, 5, 48-52.

(33) Vanucci, C.; Brusson, X.; Verdel, V.; Zana, F.; Dhomane, H.; Lhommet, G. Tetrahedron Lett. 1995, 36, 2971-2974.

(34) Yin, Y.; Zhou, H.; Sun, G.; Liu, X. J. Heterocyclic Chem. 2015, 52, 1337-1345.

(35) Schlummer, B.; Hartwig, J. F. Org. Lett. 2002,4, 1471-1474.

(36) Kim, S.; Lee, T. A.; Song, Y. Synlett 1998, 471-472.

(37) Dian, L.; Wang, S.; Zhang-Negrerie, D.; Du, Y. Adv. Synth. Catal. 2015, 357, 3836-3842.

(38) Egart, B.; Lentz, D.; Czekelius, C. J. Org. Chem. 2013, 78, 2490-2499.

(39) Amombo, O.; Marlyse, G.; Flögel, O.; Kord Daoroun Kalai, S.; Schoder, S.; Warzok, U.; Reissig, H.-U. Eur. J. Org. Chem. 2017, 1965-1972.

(40) Paterson, W.; Proctor, G. R. J. Chem. Soc. 1965, 485-489. 